\title{
FORMULATION OF THE TANGENTIAL VELOCITY SLIP PROBLEM IN TERMS OF VARIATIONAL INEQUALITIES
}

\author{
Antonio Strozzi ${ }^{1}$, Matteo Giacopini ${ }^{1}$, Enrico Bertocchi ${ }^{1}$ and Daniele Dini ${ }^{2}$ \\ ${ }^{1}$ Department of Engineering Enzo Ferrari, University of Modena and Reggio Emilia \\ ${ }^{2}$ Tribology Group, Imperial College London, South Kensington Campus
}

Corresponding author: Daniele Dini, Imperial College London, South Kensington Campus, Exhibition Rd, London SW7 2AZ, UK

Email: d.dini@imperial.ac.uk

\begin{abstract}
This contribution deals with a modelling of the tangential velocity slip problem in terms of variational inequalities. In particular, various technical situations for which the slippage problem appears to play an important role are first reviewed. Then, a mathematical formulation in terms of variational inequalities is developed where the critical shear stress criterion is considered. The theoretical conditions under which a unique solution exists are also discussed and an algebraic description based upon a complementarity approach is presented. Preliminary numerical results end the paper and a validation versus an analytical solution is proposed.
\end{abstract}




\section{Keywords}

Slip problem, critical shear stress, Tresca condition, variational inequality, complementarity problem.

\section{NOTATION}

$a$ lubricated contact length

$h$ film thickness

$h^{*}$ non-dimensional film thickness $=h / h_{\text {out }}$

$h_{\text {in }}$ film thickness at the bearing inlet

$h_{\text {out }}$ film thickness at the bearing outlet

$K$ bearing slope $=h_{\text {in }} / h_{\text {out }}-1$

$p$ fluid pressure

$p^{*}$ non-dimensional fluid pressure $=p\left(h_{\text {out }}^{2} / U^{+} / \mu / a\right)$

$p_{0}$ pressure at the bearing inlet

$p_{a}$ pressure at the bearing outlet

$q_{x}$ fluid flow in the $x$ direction

$q_{y}$ fluid flow in the $y$ direction

$u$ fluid velocity

$U^{+}$velocity of lower surface in the $x$ direction

$U$ fluid velocity at the slipping surface in the $x$ direction 
$U^{*}$ non-dimensional fluid velocity at the slipping surface in the $x$ direction $=U / U^{+}$ $x$ coordinate in the sliding direction

$x^{*}$ non-dimensional coordinate in the sliding direction $=x / a$

$y$ coordinate transverse to the sliding direction

$z$ coordinate through the film thickness

$\mu$ lubricant dynamic viscosity

$\tau$ shear stress at the slipping surface

$\tau_{\mathrm{c}}$ critical shear stress for slip in one-dimensional case

$\tau_{\mathrm{c}}{ }^{*}$ non-dimensional critical shear stress for slip in one-dimensional case $=\tau_{\mathrm{c}}\left(h_{\mathrm{ou}} / U^{+} / \mu\right)$

$\tau_{\text {cx }}$ critical shear stress for slip in the $x$ direction

$\tau_{\mathrm{cy}}$ critical shear stress for slip in the $y$ direction

\section{Introduction}

The "no-slip" assumption, i.e., the fact that the immediate layer of liquid next to a sliding surface moves with the same tangential velocity as the surface itself, is commonly employed when analysing fluid film lubrication problems and it provides boundary conditions which the derivation of the classical Reynolds equation is based on. However, the possibility of a slippage between a sliding wall and the layer of fluid in contact with it is being currently admitted for an increasing variety of technical situations. 
For example, it is known that the fluid layer in contact with the porous wall may not adhere to the wall itself in gas lubricated porous bearings [1]. A similar assumption is followed for oil lubricated, hard elastohydrodynamic contacts in [2] and it has been also admitted for soft elastohydrodynamic contacts, as are those concerning reciprocating elastomeric seals [3].

That the slippage may occur at the fluid-sealing profile interface is suggested by disagreements encountered between theoretical and experimental results.

For example, when a pressurized gas is sealed by elastomeric seals, some leakage may occur through cracks grown in the elastomer or along the narrow gaps caused by an incomplete infilling of the seal elastomer into the counterface asperities. Such a leak is particularly dangerous when the sealed gas is contaminated. It has been shown that the experimental gas flow through a narrow gap correlates poorly with the theoretical predictions computed according to the classical adhesion assumptions [4]. In this case, the tangential velocity slip assumption may partially account for the disagreement between experimental results and theoretical predictions.

Other experimental evidences exist based for examples on capillary flow measurements $[5,6]$. These studies essentially show that the rate of flow of liquids through capillaries can exceed conventional hydrodynamic theory if the liquid and the capillary surfaces are chosen so that the fluid only marginally wets the solid, i.e. the solid surface is lyophobic. 
In $[7,8]$ the authors found evidences of slip of liquid under simple Couette shear. They showed that, when the solid surface was treated to render it lyophobic, a more rapid flow of liquid adjacent to the surface was detected.

In $[9,10]$ results on the evaluation of the hydrodynamic squeeze force between a pair of crossed mica cylinders lubricated by both tetradecane and water are presented. Authors showed that, when the mica was rendered lyophobic, the squeeze force was reduced by more than one order of magnitude.

It is well known that some particular liquid such as polymer melts show actual slip at solid surfaces, related to a reduction of the viscosity of the layer of fluid close to the surface, e.g. $[11,12]$. In these cases, the slip problem has been successfully modelled by the so called Navier slip length model [13]. This model essentially relates the velocity at which the liquid slips to the shear stress arising at the interface between the fluid and the solid surface, and it treats the liquid as flowing conventionally against a fictitious surface one "slip length" below the actual surface.

This model has been applied also for mimicking the tangential slip problem in Newtonian liquids. Some researchers succeeded in fitting experimental and numerical results with flow rate independent values of the slip length $[7,8,14]$, while other scientists found that flow rate dependent values of the slip length have to be introduced $[9,15]$. 
Other models have been then proposed such as the critical shear stress model favoured for example in [2] and [16] and recently employed by Spikes in [17]. This model implies that, so long as the shear stress does not reach a critical value $\tau_{\mathrm{c}}$ anywhere within the contact zone, slip does not occur, but whenever $\tau_{\mathrm{c}}$ is reached, slip takes place at that location to maintain the surface shear stress at $\tau_{\mathrm{c}}$. In the pertinent literature, the critical shear stress condition is also referred to as Tresca condition, e.g. reference [18]. Recently a mixed formulation has been presented by Spikes and Granick [19], in which both the critical shear stress and the constant slip length criteria have been incorporated in a single model.

This contribution deals with a modelling of the tangential velocity slip problem. It is an extension of reference [16] in which the slippage problem has been formulated in terms of complementarity. In particular, in this paper a variational formulation of the slippage problem is proposed and the possible slippage in the two opposite directions is included. In the last decade, several lubrication problems have been revised in terms of variational approaches, thus retrieving information on the existence of analytical solutions and deriving suggestions on the selection of suitable numerical techniques to be employed to solve the problems. For instance, variational inequalities have been used in the description of the cavitation problem. In particular, in [20] hydrodynamic conjunctions have been considered, while in [21] elastohydrodynamic situations have been analysed. In addition, an extended variational formulation with local minimum properties has 
been proposed in [22] for the classical elastohydrodynamic lubrication problem. In [23] existence and uniqueness of the solution of the cavitation problems follow by a rigorous mathematical analysis. Recently a mass-conserving complementarity formulation of the cavitation problem has been presented by Giacopini and co-workers [24].

The present paper is organized as follows. A derivation of the basic equations governing the tangential velocity slip problem is presented in Section 2. Section 3 formulates the problem in terms of variational inequalities. The physical behaviour of the fluid is mimicked with reference to the critical shear stress model. Conditions for the existence and uniqueness of the solution are investigated. Following this, an algebraic description of the functional problem is presented that results in a definition of a linear complementarity problem. Some preliminary numerical examples end the paper and a validation versus an analytical solution is proposed.

\section{Basic equations}

Consider the Reynolds equation for quasi-stationary, unidimensional, isothermal, isoviscous, and hydrodynamic problem:

$$
\frac{\partial}{\partial x}\left(\frac{h^{3}}{6 \mu} \frac{\partial p}{\mathrm{~d} x}-U h\right)=0
$$

In the case of adhesion assumption, $U$ denotes both the velocity of the sliding profile and the velocity of the fluid layer in contact with it. In this paper, in which the 
possibility of a slippage is considered, $U^{+}$is introduced to symbolize the velocity of the sliding profile while $U$ only denotes the velocity of the fluid layer in contact with it. In addition, $p$ is the fluid pressure, $h$ is the film thickness (supposed known), and $\mu$ is the fluid viscosity.

Proper boundary conditions can be coupled with equation (1):

$p(0)=p_{0} \quad ; \quad p(a)=p_{a}$

where $a$ is the lubricated contact length.

In the interest of greater simplicity, it is assumed that a slippage can occur only between one of the sealing surfaces, namely that defined by $z=0$, and the fluid. This assumption possibly holds for a reciprocating elastomeric seal, for which the slippage is conjectured to occur between layer and elastomer [3]. This assumption can be removed at the cost of increasing theoretical difficulties.

The following manipulations aim at expressing the fluid shear stress, $\tau$, at the fluid layer contacting the sliding profile, as a function of the velocity of the fluid layer in contact with the sliding surface, $U$. Figure 1 shows the schematic of a classical hydrodynamic bearing and helps at clarifying the geometry of the problem and the meaning of the main variables involved. 


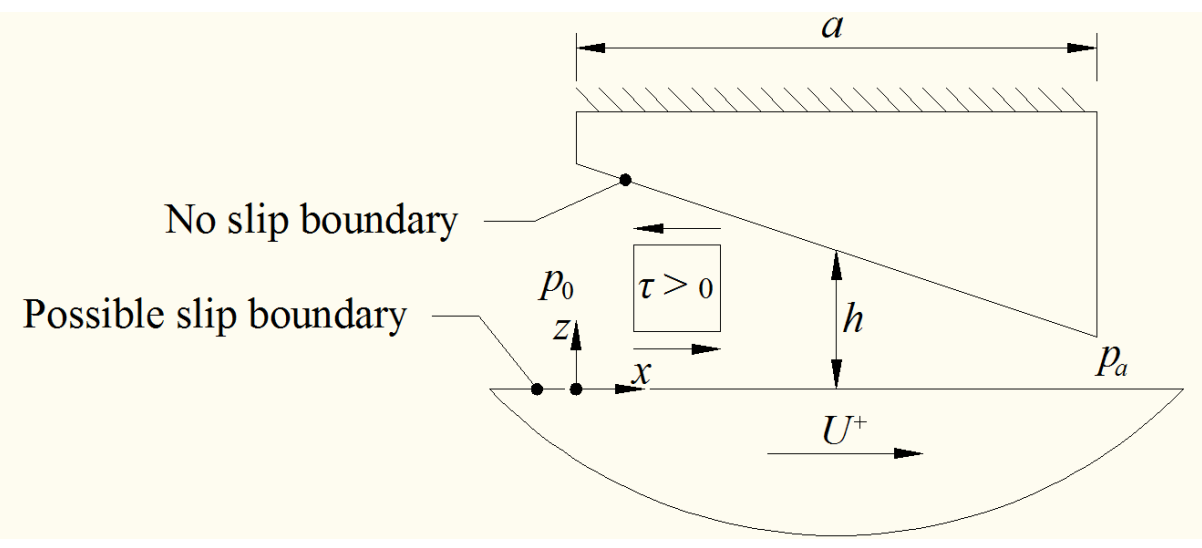

Figure 1. The meaning of main symbols adopted for the tangential velocity slip problem.

By integrating twice equation (1) and imposing the boundary conditions (2), the expression of the fluid pressure, $p$, as a function of $U$ is derived:

$$
p(x)=6 \mu \int_{0}^{x} \frac{U}{h^{2}} d y+\left[\left(p_{a}-p_{0}\right)-6 \mu \int_{0}^{a} \frac{U}{h^{2}} d y\right] \frac{\int_{0}^{x} \frac{1}{h^{3}} d y}{\int_{0}^{a} \frac{1}{h^{3}} d y}+p_{0}
$$

Referring to Figure 1 for the convention on signs, the shear stress within the fluid in contact with the sliding profile $\tau$, i.e. for $z=0$, is (see the derivation of equation (B5) in the Appendix B):

$$
\left.\tau\right|_{z=0}=\frac{h}{2} \frac{d p}{d x}+U \frac{\mu}{h}
$$

Equation (4) agrees with the physical reasoning presented in [17] on the sign of the shear stress. In fact, from the physical point of view, when a slippage occurs between the fluid and a solid surface, the shear stress at the interface will always tend to resist 
slip against the surface and so it is natural to consider it as positive if the fluid velocity relative to the wall is negative and vice versa.

By substituting equation (3) into equation (4) one obtains:

$$
\left.\tau\right|_{z=0}=4 \mu \frac{U}{h}-3 \frac{\mu}{h^{2}} \frac{\int_{0}^{a} \frac{U}{h^{2}} d y}{\int_{0}^{a} \frac{1}{h^{3}} d y}+\frac{p_{a}-p_{0}}{2 h^{2} \int_{0}^{a} \frac{1}{h^{3}} d y}
$$

This functional expression can be given a compact form:

$$
\tau=L U+Q
$$

where $L$ and $Q$ are identified by comparing equation (5) with equation (6).

\section{Formulation in terms of variational inequalities}

In this section the formulation of the tangential velocity slip problem in terms of variational inequalities is derived. In particular, the critical shear stress criterion is employed in order to mimic the slippage between the fluid and the solid surfaces. It is clarified that the variational formulation accounts for the possible slippage in the two opposite directions whereas the preliminary work [16] considered slippage only in one direction. An advantage of the variational formulation with respect to the complementarity approach of [16] is that it naturally incorporates the possibility of slippage in the two opposite directions since it is relatively easy to consider a finite 
interval for the values assumed by the shear stress at the slippage surface, $\tau$. An example including slippage in both directions is presented in Figure 3.

In detail, the physical significance of the problem under examination requires that, if the shear stress $\tau$ is internal to the critical values $\tau_{\mathrm{c}}{ }^{-}, \tau_{\mathrm{c}}{ }^{+}$(where usually $\tau_{\mathrm{c}}{ }^{-}=-\tau_{\mathrm{c}}{ }^{+}$), no slippage occurs, and therefore $U=U^{+}$. If $\tau$ reaches its critical upper bound $\tau_{\mathrm{c}}^{+}$, the fluid velocity can be lower than that of the sliding profile, $U \leq U^{+}$. Finally, if $\tau=\tau_{\mathrm{c}}^{-}, U \geq U^{+}$. In summary:

$\tau \in T$

$T=\left\{\tau ; \tau_{c}^{-} \leq \tau \leq \tau_{c}^{+}\right\}$

where $T$ is a closed convex set. Moreover it will be shown in the following that the variational inequality must hold true:

$\left(U-U^{+}, \sigma-\tau\right) \geq 0 \quad \tau \in T, \quad \forall \sigma \in T$

The meaning of the variational inequality (9) can be illustrated via a simple algebraic example. In fact, if $\tau$ is internal to the segment $\tau_{c}^{-}, \tau_{c}^{+}, U$ must coincide with $U^{+}$ according to physical reasons. Since $\sigma$ can be chosen greater or smaller than $\tau,(\sigma-\tau)$ can be positive, negative or null. Consequently, inequality (9) requires that $\left(U-U^{+}\right)$ vanishes, that is $U=U^{+}$.

Conversely, if $\tau=\tau_{\mathrm{c}}{ }^{+},(\sigma-\tau)$ can be negative or null, but not positive, and consequently $U \leq U^{+}$ 
Finally, if $\tau=\tau_{\mathrm{c}}^{-},(\sigma-\tau) \geq 0$ and $U \geq U^{+}$.

These aspects are well explained in [25], to which the interested reader is referred.

In [2] the slippage problem was modelled analysing various distinct zones. The variational inequality approach permits such zones to be unified under the same modelling.

Once the variational inequality governing the problem is defined, the functional connection between the two variables of the inner product of inequality (9) can be easily identified manipulating equation (6):

$$
\begin{aligned}
& \tau=L U+Q \Rightarrow U=L^{-1} \tau-L^{-1} Q \\
& U-U^{+}=L^{-1} \tau-L^{-1} Q-U^{+} \Rightarrow U-U^{+}=M \tau+P
\end{aligned}
$$

where operator $M$ of equation (11) equals the inverse of $L$ appearing in equation (6).

In conclusion, the tangential velocity slip problem can be expressed in the following way:

Find $\tau \in T, \quad T=\left\{\tau ; \tau_{c}^{-} \leq \tau \leq \tau_{c}^{+}\right\}$, such that:

(i) $U-U^{+}=M \tau+P$

(ii) $\left(U-U^{+}, \sigma-\tau\right) \geq 0 \quad \tau \in T, \quad \forall \sigma \in T$

In the following, considerations about existence and uniqueness of the solution of the problem expressed in equations (12) and (13) are presented. To this aim, some properties of the operators $L$ and $M$ of equations (6) and (11) have to be investigated. As 
a result of the mathematical passages described in detail in the Appendix A the solution exists and is unique, since $T$ is a closed convex set and $M$, in the limits of condition (A15), is coercive (that is, $(M \tau, \tau) \geq \alpha\|\tau\|^{2}$, see the Appendix A), e.g. [26]. In other words, once the profile, the sliding velocity and the fluid viscosity have been chosen, the adhesion and the slippage zones are unequivocally determined. Beyond its unifying features, the variational inequality approach supplies basic information about existence and uniqueness of the solution. This conclusion does not necessarily imply that such zones can be easily located, nor does it provide a constructive way for achieving closedform solutions. However, it naturally lead to the development of linear complementarity routines that locate the solution in a finite number of steps.

The next section presents a reformulation of the tangential velocity slip problem, which is suitable for an algebraic description. This formulation is borrowed from the area of elastic-plastic and unilateral contact studies [27].

\section{Algebraic description}

The properties demonstrated in the previous section provide a sound mathematical basis for an algebraic description of the problem expressed in equations (12) and (13).

Equation (12) can be written as:

$$
M \tau+\left(U^{+}-U\right)=-P
$$


In order to describe the problem in terms of complementarity, two different complementarity conditions have to be considered that are related to the physical meaning of inequality (13). In fact, if $\tau$ reaches its upper bound $\tau_{\mathrm{c}}^{+}$, the velocity of the fluid in contact with the sliding profile $U$, becomes lower than the profile velocity $U^{+}$, making the problem governed by the two complementary variable $\left(\tau_{\mathrm{c}}^{+}-\tau\right)$ and $\left(U^{+}-\right.$ $U)$. On the other hand, if $\tau$ reaches its lower bound $\tau_{\mathrm{c}}^{-}$, the velocity of the fluid in contact with the sliding profile $U$, becomes greater than the profile velocity $U^{+}$, and the two complementary variables to be considered are $\left(\tau-\tau_{\mathrm{c}}^{-}\right)$and $\left(U-U^{+}\right)$.

The problem is similar to the one connected with the analysis of frictional contact problems solved via complementarity methods [28] in which methods have been presented to simultaneously handle several pairs of complementary variables. Similarly, suitable auxiliary variables $\Delta U^{-}$and $\Delta U^{+}$can be introduced for the slip problem such that:

$U=U^{+}-\Delta U^{+}+\Delta U^{-}$

with:

$$
\begin{aligned}
& \Delta U^{+}=\max \left(U^{+}-U, 0\right) \\
& \Delta U^{-}=\max \left(U-U^{+}, 0\right)
\end{aligned}
$$


It will be shown at the end of the paragraph that the orthogonality condition between $\Delta U^{-}$and $\Delta U^{+}$will be automatically satisfied once the complementarity problem has been derived.

By introducing $\underline{N}$ :

$\underline{N}^{T}=\left[\begin{array}{ll}+1 & -1\end{array}\right]$

and $\underline{\Delta U^{T}}$ :

$\underline{\Delta U^{T}}=\left[\begin{array}{ll}\Delta U^{+} & \Delta U^{-}\end{array}\right]$

equation (14) is rewritten as:

$M \tau+\underline{N}^{T} \underline{\Delta U}=-P$

Also, by introducing vector $\underline{\tau}^{*}$ :

$\underline{\tau}^{*^{T}}=\left[\begin{array}{ll}\tau_{c}^{+} & -\tau_{c}^{-}\end{array}\right]$

the inequality expressed in (8) can be rewritten as:

$\underline{N \tau} \leq \underline{\tau}^{*}$

Finally, condition (19) and (21) can be given an operatorial description according to the following tableau:

$\left[\begin{array}{cc}M & \underline{N}^{T} \\ \underline{N} & 0\end{array}\right]\left[\begin{array}{c}\tau \\ \Delta U\end{array}\right] \leq\left[\begin{array}{c}-P \\ \underline{\tau}^{*}\end{array}\right]$ 
The symmetrical system (22) can be condensed at an analytical level, to produce a unique functional equation. To this aim, $\tau$ is expressed in terms of $\underline{\Delta U}$ by employing equation (19):

$$
\tau=-M^{-1}\left(\underline{N}^{T} \underline{\Delta U}+P\right)
$$

or, by using inequality (21):

$$
\underline{N} M^{-1}\left(\underline{N}^{T} \underline{\Delta U}+P\right)+\underline{\tau}^{*}=\underline{\Delta \tau} \geq 0
$$

where $\underline{\Delta \tau}$ is the slack variable connected with inequality (21):

$$
\begin{aligned}
& \Delta \tau^{+}=\tau_{c}^{+}-\tau \\
& \Delta \tau^{-}=\tau-\tau_{c}^{-} \\
& \Delta \tau^{T}=\left[\begin{array}{ll}
\Delta \tau^{+} & \Delta \tau^{-}
\end{array}\right]
\end{aligned}
$$

The whole problem can finally be stated via a typical complementarity approach as follows:

$\underline{N} M^{-1} \underline{N}^{T} \underline{\Delta U}+\underline{N} M^{-1} P+\underline{\tau}^{*}=\underline{\Delta \tau}$

$\underline{\Delta \tau} \geq 0$

$\underline{\Delta U} \geq 0$

$\underline{\Delta U^{T}} \underline{\Delta \tau}=0$

In fact, if $\tau$ equals $\tau_{\mathrm{c}}^{+}$, the first entry of $\underline{\Delta \tau}$ vanishes, while the second entry of $\underline{\Delta \tau}$ equals $\left(\tau_{\mathrm{c}}^{+}-\tau_{\mathrm{c}}{ }^{-}\right)$, and therefore it is positive. The sign restriction (28) imposes $\underline{\Delta U} \geq 0$. Because 
of the orthogonality condition (29), the first entry of $\underline{\Delta U}, \Delta U^{+}$, can be positive or null, while the second entry, $\Delta U^{-}$, must vanish. Thus, if $\tau$ reaches its upper critical value $\tau_{\mathrm{c}}{ }^{+}$, then $\Delta U^{+} \geq 0$ and so $U \leq U^{+}$.

Similarly, if $\tau$ equals $\tau_{c}^{-}$, the second entry of $\underline{\Delta \tau}$ vanishes, while the first entry equals $\left(\tau_{\mathrm{c}}^{+}-\tau_{\mathrm{c}}^{-}\right)$. Condition (29) requires that the first entry of $\underline{\Delta U}, \Delta U^{+}$, vanishes, while the second entry, $\Delta U^{-}$, can be positive or null. Thus, if $\tau$ reaches its lower bound $\tau_{\mathrm{c}}^{-}$, then $\Delta U^{-} \geq 0$ and so $U \geq U^{+}$.

Finally, if both entries of $\underline{\Delta \tau}$ are positive, equation (29) implies that $\underline{\Delta U}$ vanishes, and so both $\Delta U^{+}$and $\Delta U^{-}$are null. According to this situation, if the shear stress does not reach its critical bounds, no slippage occurs, and $U=U^{+}$.

In conclusion, it has been shown that, as it was anticipated at the beginning of this section, at most one of the two variables $\Delta U^{-}, \Delta U^{+}$does not vanish, which implies that $\Delta U^{-}$and $\Delta U^{+}$are orthogonal variables.

The linear operator acting on $\underline{\Delta U}$ in equation (26) is coercive with respect to $\underline{N}^{\mathrm{T}} \underline{\Delta U}$. In fact, since $M=L^{-1}$ :

$$
\left(\underline{N}^{T} \underline{\Delta U}, L \underline{N}^{T} \underline{\Delta U}\right) \geq \alpha\left\|\underline{N}^{T} \underline{\Delta U}\right\|^{2} \quad \alpha>0
$$

or after reordering:

$$
\left(\underline{\Delta U}, \underline{N} L \underline{N}^{T} \underline{\Delta U}\right) \geq \alpha\left\|\underline{N}^{T} \underline{\Delta U}\right\|^{2} \quad \alpha>0
$$


So, the term $\left(\underline{\Delta U}, \underline{N} L \underline{N}^{T} \underline{\Delta U}\right)$ is positive or null, but the operator $\underline{N} L \underline{N}^{T}$ acting on $\underline{\Delta U}$ is no more coercive, since for $\Delta U^{+}=\Delta U^{-} \neq 0, \underline{N}^{\mathrm{T}} \underline{\Delta U}=0$, and therefore:

$$
\left(\underline{\Delta U}, \underline{N} L \underline{N}^{T} \underline{\Delta U}\right)=0
$$

while:

$$
\|\underline{\Delta U}\|^{2}>0
$$

Because of the structure of $\underline{N}$, operator $\underline{N} L \underline{N}^{T}$ loses its coerciveness property only in the presence of the vectors $\underline{\Delta U}$ of the above-mentioned type $\left(\Delta U^{+}=\Delta U^{-} \neq 0\right)$. According to the previously outlined observations, the matrix deriving from the discretization of operator $\underline{N} L \underline{N}^{T}$ is positive-semidefinite. The problem expressed in equations (26) to (29) can be processed numerically via suitable pivoting complementarity routines [24, 27], which converge to the solution in a finite number of iterations if the matrix which defines the complementarity problem is positive-definite. However, in our case positive-semidefiniteness is activated only by a vector $\underline{\Delta U}$ formed by $\Delta U^{+}=\Delta U^{-} \neq 0$, a condition which cannot actually occur, as explained before. As a consequence, convergence of the complementarity routines is recovered, and, therefore, the adhesion and slippage zones are located in a finite number of iterations.

Alternatively, a solution method based on heuristics could be devised. An initial solution according to the adhesion assumption could be computed. Then, a slippage 
would be admitted for the node where the shear stress at the fluid-wall interface most exceeds the limit shear strength of the fluid. The no-slippage condition at the corresponding node could be released by imposing that in that node the shear stress equals the critical value, and consequently the velocity of the fluid contacting the wall could be determined for that node. The new shear stress distribution could be computed and the previously outlined procedure could be repeated until all the nodal shear stresses do not exceed the shear stress critical value. Similar methods where applied for example to solve cavitation problems in [29]. Contrary to the method favoured in this paper, a strategy based on heuristics does not guarantee the numerical convergence, or it might even be trapped in an endless loop. Moreover, this method would not supply any information about the existence or uniqueness of the solution.

The algebraic description of the tangential velocity slip problem favoured in this section can then be implemented with few efforts in the Finite Element framework in a similar manner as that presented in [30] where the cavitation problem has been considered and to which the interest reader is referred for details.

\section{Numerical examples}

In this section, preliminary numerical examples are presented, based on the algorithm developed in this paper. These examples aim at clarifying the salient capabilities of the numerical method rather than at examining technically realistic situations. 
In the interest of greater simplicity, a simple linear slider (Figure1) is studied.

To evidence the potentials of the proposed approach, two different sets of examples are presented; the first set considers situations in which slippage occurs only in one direction, while the second set accounts for a two direction slippage.

Table 1 collects the main geometrical and physical parameters for the first set of examples. In particular, three different situations are investigated in which all the geometrical and physical parameters governing the problem are kept constant apart from the critical shear stress of the fluid. Three different values of the critical shear stress $\tau_{\mathrm{c}}$, namely 30,40 and $50 \mathrm{~Pa}$, are considered.

Figure 2(a) shows results in terms of fluid pressure, $p$, as a function of the critical shear stress of the fluid, $\tau_{\mathrm{c}}$. As the value of the critical shear stress decreases, the maximum pressure decreases as well. Asymptotically, for the limit condition $\tau_{\mathrm{c}}=0$, no lubricant entrains the bearing profile and thus no load support exists (see equation B10 in the Appendix B). Total or partial slip against the moving lower surface seems therefore to be undesirable for the bearing performance. Practically more relevant results are presented in [31-33] where slip is considered to occur with respect to the upper fixed surface. Authors are planning to extent the application of the proposed methodology to the analysis of similar cases, including also the cavitation effect by combining the present algorithm with the mass conserving complemetarity algorithm for cavitation presented in [24]. 
Figure 2(b) depicts the shear stress for $z=0, \tau$, and the speed $U$ of the layer of fluid in contact with the sliding profile for the three different values of the critical shear stress, $\tau_{\mathrm{c}}$. Three different macroscopic fluid behaviours can be identified. In particular, when the lowest value of the critical shear stress is considered (namely $\tau_{\mathrm{c}}=30 \mathrm{~Pa}$ ) a full slip condition is encountered and, everywhere inside the bearing domain, $\tau=\tau_{\mathrm{c}}=30 \mathrm{~Pa}$ and $U<U^{+}$. When the value of the critical shear stress is increased to $40 \mathrm{~Pa}$, a partial slip condition is detected and slippage occurs only in a limited portion of the bearing domain. Finally, when the greatest value of $\tau_{\mathrm{c}}=50 \mathrm{~Pa}$ is considered, a no-slip behaviour is detected and everywhere inside the bearing domain $|\tau|<\tau_{\mathrm{c}}$ and $U=U^{+}$.

Table 1. Geometrical and physical parameters for the first set of examples.

\begin{tabular}{|l|l|l|}
\hline Film thickness at the inlet $h_{\text {in }}$ & $2 \cdot 10^{-3}$ & $\mathrm{~m}$ \\
\hline Film thickness at the outlet $h_{\text {out }}$ & $1 \mathrm{e}-3$ & $\mathrm{~m}$ \\
\hline Bearing slope $K=h_{\text {in }} / h_{\text {out }}-1$ & 1 & \\
\hline Slider length $a$ & 1 & $\mathrm{~m}$ \\
\hline Fluid pressure at the inlet $p_{0}$ & 0 & $\mathrm{~Pa}$ \\
\hline Fluid pressure at the outlet $p_{a}$ & 0 & $\mathrm{~Pa}$ \\
\hline Sliding velocity $U^{+}$ & 0.4 & $\mathrm{~m} / \mathrm{s}$ \\
\hline Lubricant viscosity $\mu$ & $125 \cdot 10^{-3}$ & $\mathrm{~Pa} \mathrm{~s}$ \\
\hline Critical shear stress $\tau_{\mathrm{c}}$ & $30 / 40 / 50$ & $\mathrm{~Pa}$ \\
\hline
\end{tabular}




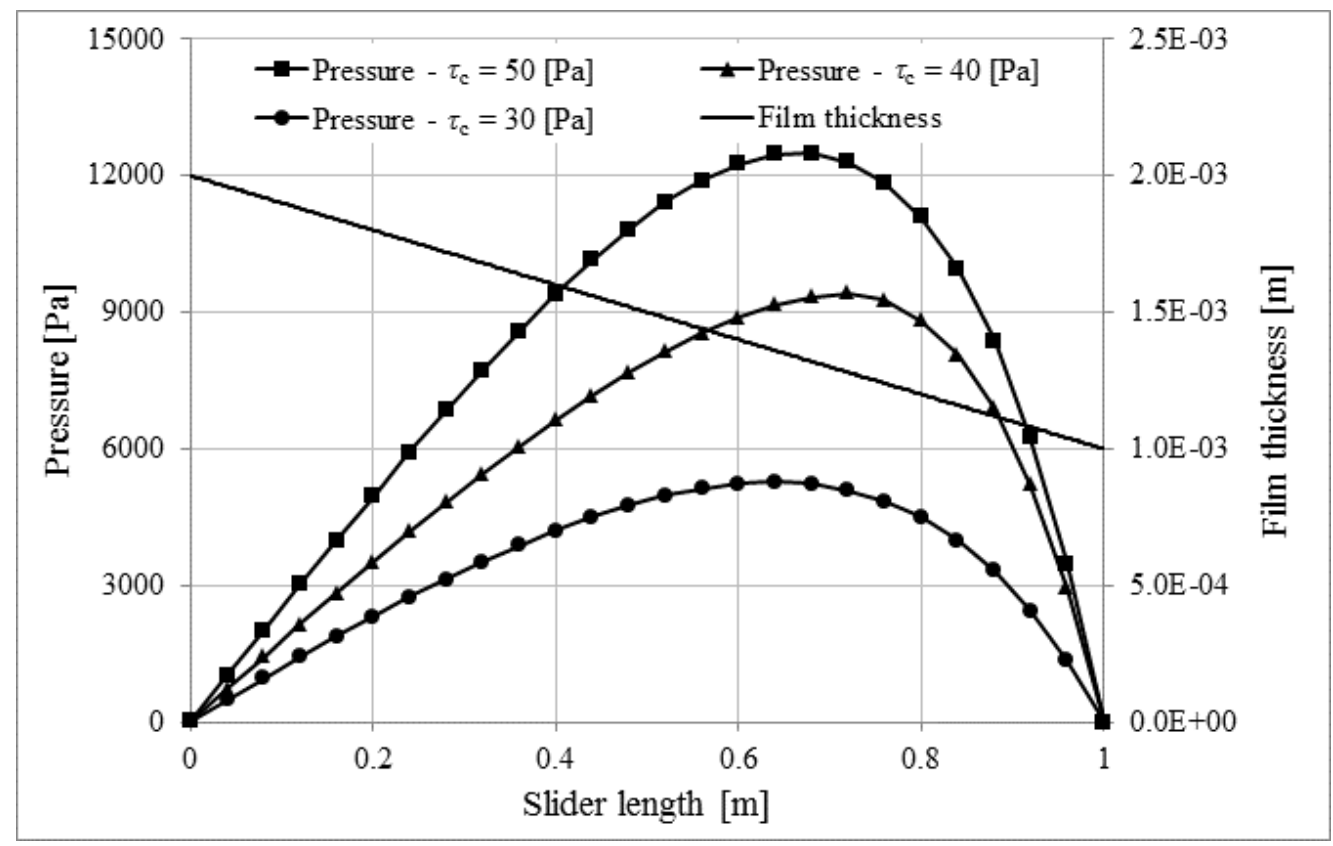

(a)

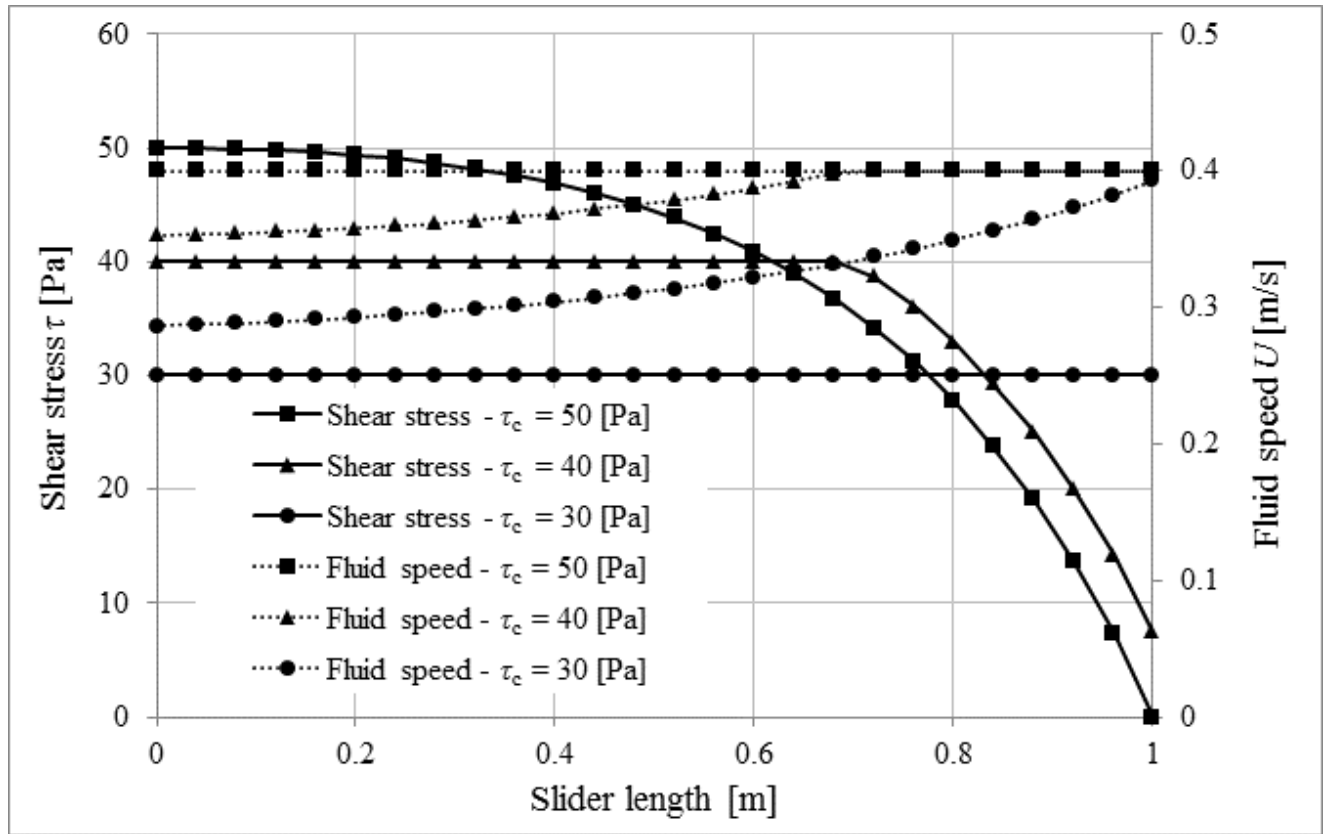

(b)

Figure 2. Fluid pressure (a), shear stress and fluid layer speed (b), for the selected cases of Table 1. 
The results presented in Figure 2 do not completely evidence the proposed algorithm capabilities. In fact, in all the three situations investigated, slip occurs only in the opposite direction of the moving profile velocity $U^{+}$, i.e. with a negative relative velocity between fluid and moving profile. In order to evidence the potentials of the proposed approach, additional selected examples have been introduced in which slip occurs also in the same direction as $U^{+}$. Such examples complete the results obtained in a previous contribution [16]. Table 2 collects the geometrical and physical parameters of this second set of cases.

Figure 3(a) shows the results in terms of fluid pressure, $p$, as a function of the critical shear stress of the fluid, $\tau_{\mathrm{c}}$, for this second set of selected cases. Again, as the value of the critical shear stress decreases, the maximum pressure decreases as well.

Figure 3(b) depicts the shear stress for $z=0$, $\tau$, and the speed $U$ of the layer of fluid in contact with the sliding profile for the three different values of the critical shear stress, $\tau_{c}$. Three different macroscopic fluid behaviours can be identified. In particular, when the highest value of the critical shear stress is considered (namely $\tau_{\mathrm{c}}=15 \mathrm{~Pa}$ ) a no-slip condition is encountered, since everywhere inside the bearing domain, $|\tau|<\tau_{\mathrm{c}}=15 \mathrm{~Pa}$ and $U=U^{+}$. When the value of the critical shear stress is decreased to $10 \mathrm{~Pa}$, a partial slip condition is detected in which slippage occurs forwards in the vicinity of the outlet, where the velocity $U$ of the fluid in contact with the sliding profile is higher than the profile velocity $U^{+}$. Finally, when the lowest value of $\tau_{\mathrm{c}}=8 \mathrm{~Pa}$ is considered, a partial, 
more complex slip behaviour is detected. In particular, in the central part of the bearing, slippage occurs backwards with the velocity $U$ of the fluid in contact with the sliding profile lower than the profile velocity $U^{+}$; in the vicinity of the outlet, slippage occurs forwards, with the velocity $U$ of the fluid in contact with the sliding profile higher than the profile velocity $U^{+}$.

Table 2. Geometrical and physical parameters for the second set of examples.

\begin{tabular}{|l|l|l|}
\hline Film thickness at the inlet $h_{\text {in }}$ & $6 \cdot 10^{-3}$ & $\mathrm{~m}$ \\
\hline Film thickness at the outlet $h_{\text {out }}$ & $1 \mathrm{e}-3$ & $\mathrm{~m}$ \\
\hline Bearing slope $K=h_{\text {in }} / h_{\text {out }}-1$ & 5 & \\
\hline Slider length $a$ & 1 & $\mathrm{~m}$ \\
\hline Fluid pressure at the inlet $p_{0}$ & 0 & $\mathrm{~Pa}$ \\
\hline Fluid pressure at the outlet $p_{a}$ & 0 & $\mathrm{~Pa}$ \\
\hline Sliding velocity $U^{+}$ & 0.1 & $\mathrm{~m} / \mathrm{s}$ \\
\hline Lubricant viscosity $\mu$ & $125 \cdot 10^{-3}$ & $\mathrm{~Pa} \mathrm{~s}$ \\
\hline Critical shear stress $\tau_{\mathrm{c}}$ & $8 / 10 / 15$ & $\mathrm{~Pa}$ \\
\hline
\end{tabular}




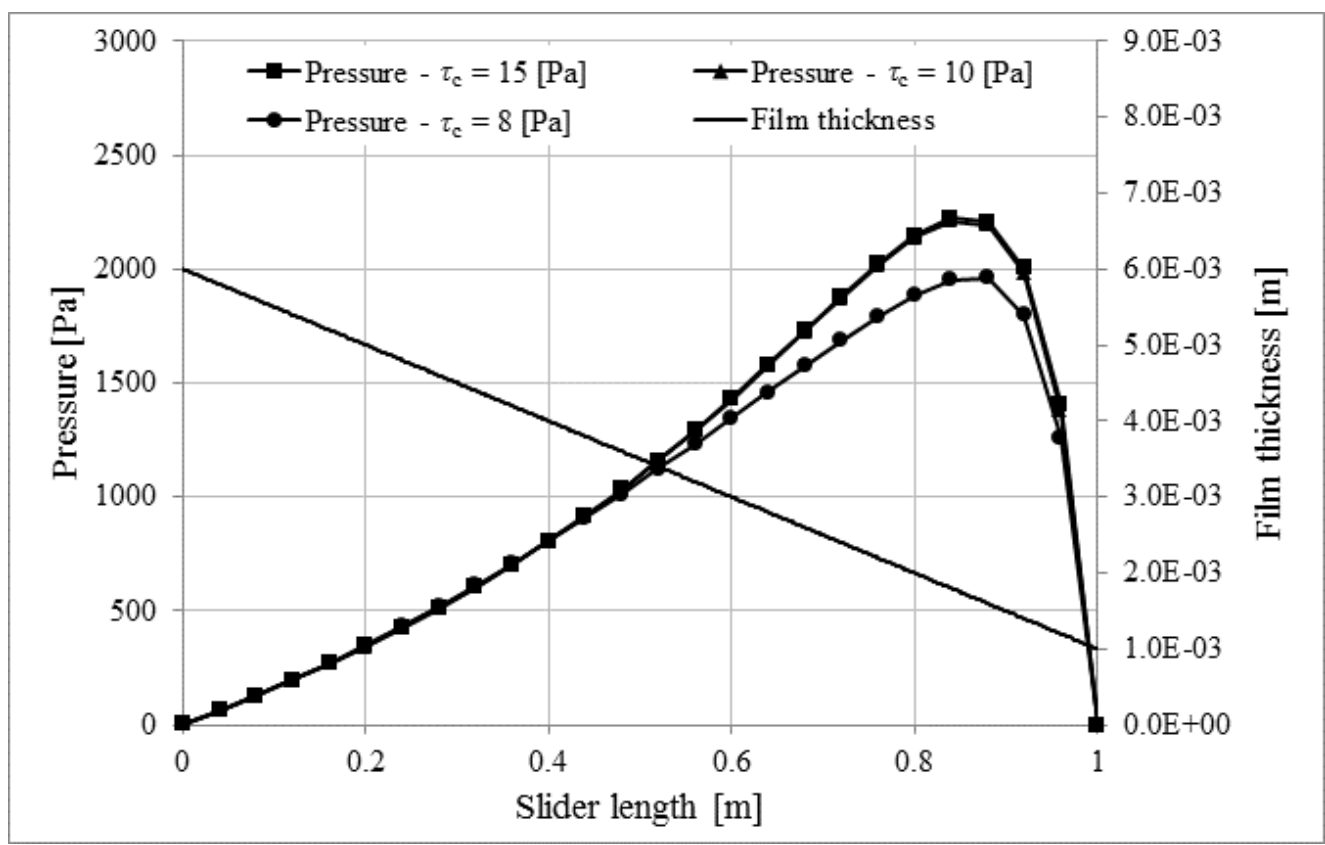

(a)

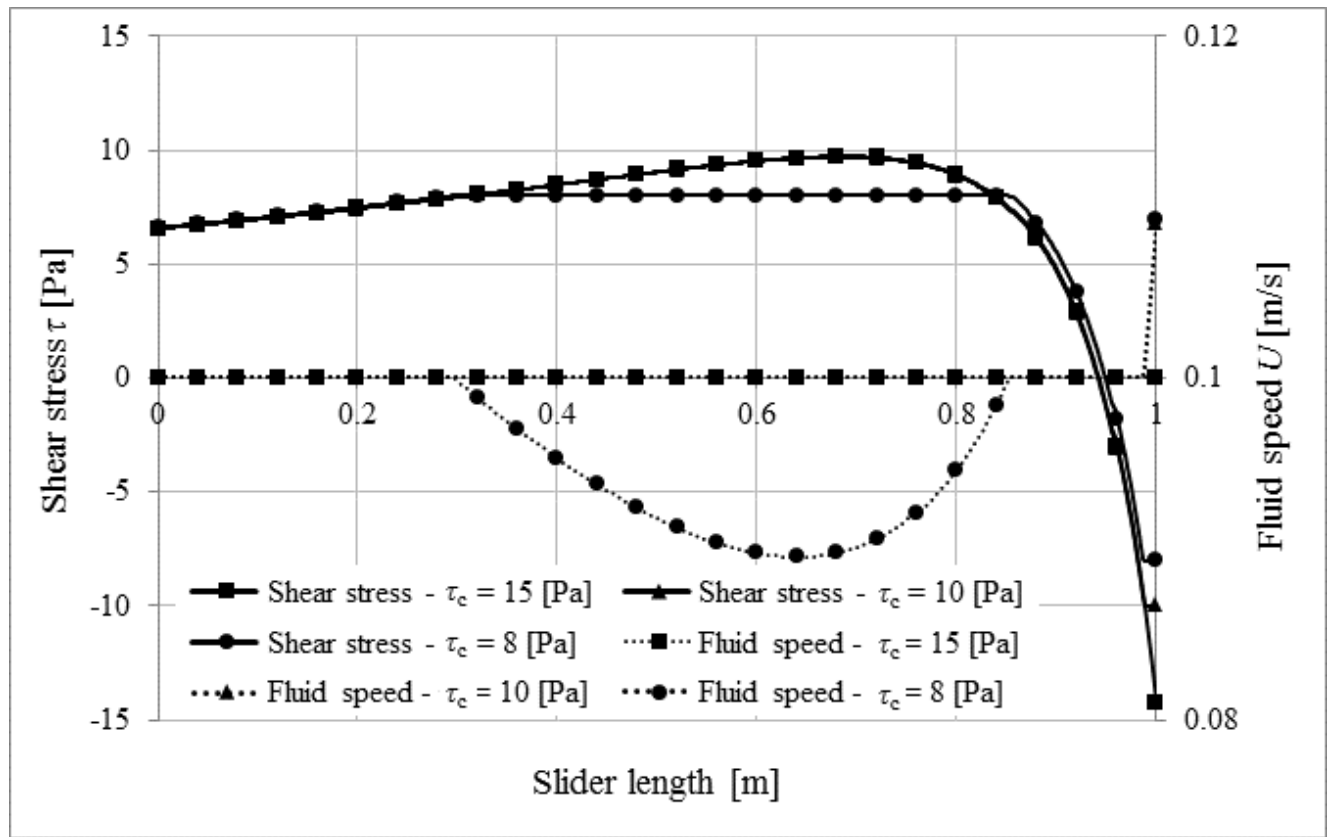

(b)

Figure 3. Fluid pressure (a), shear stress and fluid layer speed (b), for the selected cases of Table 2. 
Once the capabilities of the algorithm developed have been tested via these simple discrete examples, a numerical campaign has then been carried out in order to identify the fluid behaviour for wide intervals of the critical shear stress of the fluid, $\tau_{\mathrm{c}}$, and of the bearing slope $K$. Figure 4 shows a map where results are presented in a nondimensional form considering the non-dimensional critical shear stress, $\tau_{\mathrm{c}}{ }^{*}=\tau_{\mathrm{c}}$ $\left(h_{\text {out }} / U^{+} / \mu\right)$. The three different sliding zones, namely no-slip zone, partial slip zone and full slip zone, are identified. In particular, the upper bound of the partial slip zone is described by a seagull wing profile; the left (right) wing addresses a backwards (forwards) slip. For $K>\Phi-1=(\sqrt{5}+1) / 2-1 \approx 0.618$ it is no longer possible to demonstrate that the operator $M$ of equation (11) is coercive, see condition (A15) of the Appendix A. Despite this limit, the numerical routines successfully detect the slip and no-slip zones.

Figure 4 of the present paper represents a sort of counterpart of Figure 3 of [17] where slip has been supposed to occur only against the upper fixed surface of the same simple converging bearing.

The results of Figure 4 have been validated versus two analytical solutions capable to describe the upper and lower bounds of the partial slip zone. The derivation of the equations employed is presented in detail in the Appendix B. Numerical and analytical results basically coincide (see Figure 5) thus providing a benchmark for the algorithm developed. A mesh size sensitivity analysis has been carried out for all the 
configurations of Figure 4; this analysis has shown that a number of equispaced elements higher than two hundred always provides accurate results.

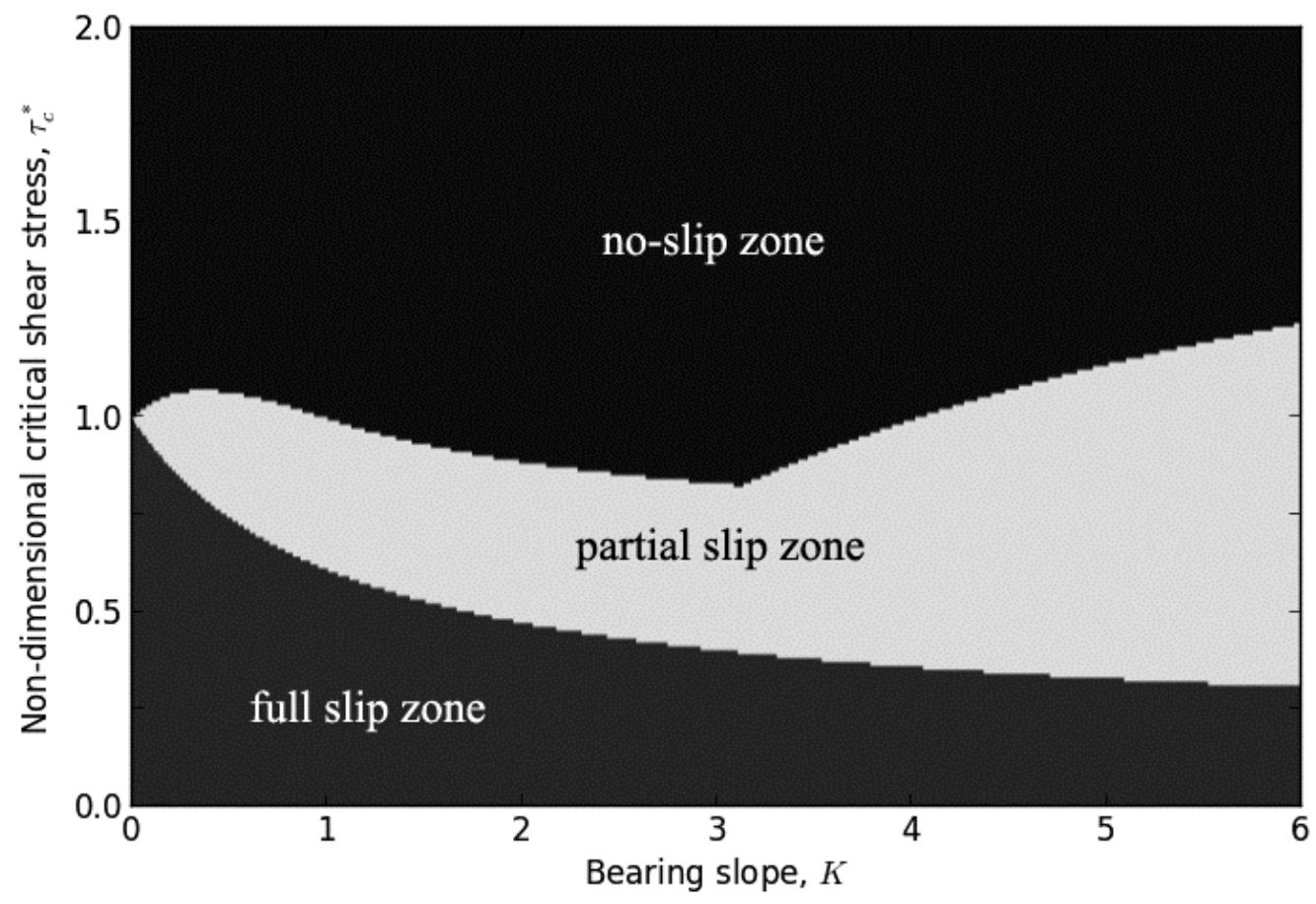

Figure 4. Map of the contact slip conditions in a convergent slider.

\section{Conclusions}

In the present paper, the tangential velocity slip problem has been formulated in terms of variational inequalities. The theoretical conditions under which a unique solution exists have been discussed. A formulation suitable for an algebraic description resulting in a linear complementarity problem has been developed. Based upon the previous point, a Finite Element algorithm has been implemented and suitable pivoting routines 
have been employed in order to find a solution of the problem in a finite number of iterations. Some preliminary numerical examples have been finally presented, aimed at illustrating the salient capabilities of the method. The numerical results have been assessed versus purposely developed analytical solutions.

\section{Bibliography}

[1] Malik M and Rodkiewicz Cz M. On slip flow considerations in gas-lubricated porous bearings. J Tribol-T Asme 1984; 106: 484-491.

[2] Jacobson BO and Hamrok BJ. Non-newtonian fluid model incorporated into elastohydrodynamic lubrication of rectangular contacts. J Tribol-T Asme 1984; 106: $275-284$.

[3] Theyse FH. The inverse hydrodynamic theory and its application in the design of controlled leakage seals between moving parts. In: Proceedings of the Third International Conference on Fluid Sealing, Cambridge, England, 1967, pp.F2/17-32. Cranfield, British Hydromechanics Research Association.

[4] George AF and Williams D. An investigation of leakage past static elastomeric seals. In: Papers presented at the Tenth International Conference on Fluid Sealing, Innsbruck, Austria, April 3-5 1983, pp.43-53. Cranfield, British Hydromechanics Research Association. 
[5] Churaev NV, Sobolev VD and Somov AN. Slippage of Liquids over Lyophobic Solid Surfaces. J Colloid Interf Sci 1984; 97: 574-581.

[6] Vinogradova OI. Slippage of water over hydrophobic surfaces. Int J Miner Process 1999; 56: 31-60.

[7] Pit R, Hervet H and Leger L. Friction and slip of a simple liquid at a solid surface. Tribol Lett 1999; 7: 147-152.

[8] Pit R, Hervet H and Leger L. Direct Experimental Evidence of Slip in Hexadecane: Solid Interfaces. Phys Rev Lett 2000; 85: 980-983.

[9] Zhu Y and Granick S. Rate-Dependent Slip of Newtonian Liquid at Smooth Surfaces. Phys Rev Lett 2001; 87: 096105-1-4.

[10] Zhu Y and Granick S. Limits of the Hydrodynamic No-Slip Boundary Condition. Phys Rev Lett 2002; 88: 106102-1-4.

[11] Léger L, Raphael E and Hervet H. Surface-Anchored Polymer Chains: Their Role in Adhesion and Friction. Adv Polym Sci 1999; 138: 185-225.

[12] Wang SQ. Molecular Transitions and Dynamics at Polymer / Wall Interfaces: Origins of Flow Instabilities and Wall Slip. Adv Polym Sci 1999; 138: 227-275.

[13] Vinogradova OI. Drainage of a Thin Liquid Film Confined between Hydrophobic Surfaces. Langmuir 1995; 11: 2213-2220. 
[14] Bonaccurso E, Kappl M and Butt HJ. Hydrodynamic Force Measurements: Boundary Slip of Water on Hydrophilic Surfaces and Electrokinetic Effects. Phys Rev Lett 2002; 88: 076103-1-4.

[15] Zhu Y and Granick S. No-Slip Boundary Condition Switches to Partial Slip When Fluid Contains Surfactant. Langmuir 2002; 18: 10058-10063.

[16] Strozzi A. Formulation of three lubrication problems in terms of complementarity. Wear 1985; 104: 103-119.

[17] Spikes H. The half-wetted bearing. Part 1: extended Reynolds equation. P I Mech Eng J-J-Eng 2003; 217(1): 1-14.

[18] Bayada G. and Boukrouche M. On a free boundary problem for the Reynolds equation derived from the Stokes system with Tresca boundary conditions. J Math Anal Appl 2003; 282: 212-231.

[19] Spikes H and Granick S. Equation for slip of simple liquids at smooth solid surfaces. Langmuir 2003; 19: 5065-5071.

[20] Rhode SM and McAllister GT. A variational formulation for a class of free boundary problems arising in hydrodynamic lubrication. Int J Eng Sci 1975; 13: 841850.

[21] Oden JT and Wu SR. Existence of solutions to the Reynolds' equation of elastohydrodynamic lubrication. Int J Eng Sci 1985; 23: 207-215. 
[22] Strozzi A. The elastohydrodynamic problem expressed in terms of extended variational formulation. J Tribol-T Asme 1986; 108: 557-564.

[23] Bayada G, Talibi MEA, Hadi KA. Existence and uniqueness for a non-coercive lubrication problem, J Math Anal Appl 2007; 327: 585-610.

[24] Giacopini M, Fowell MT, Dini D, et al. A Mass-Conserving Complementarity Formulation to Study Lubricant Films in the Presence of Cavitation, J Tribol-T Asme 2010; 132(4): 041702.

[25] Oden JT and Kikuci NK. Theory of variational inequalities with applications to problem of flow through porous media. Int J Eng Sci 1980; 18(10): 1173-1284.

[26] Lions JT. Partial differential inequalities. Russ Math Surv+ 1972; 27(2): 91-159.

[27] Cannarozzi AA. On the resolution of some unilaterally constrained problems in structural engineering. Computer Method Appl Mech Eng 1980; 24: 339-357.

[28] Funk K and Pfeiffer F. A time-stepping algorithm for stiff mechanical systems with unilateral constraints. PAMM - Proc Appl Math Mech 2003; 2: 228-229.

[29] Optasanu V and Bonneau D. Finite Element Mass-Conserving Cavitation Algorithm in Pure Squeeze Motion. Validation/Application to a Connecting- Rod Small End Bearing. J Tribol-T Asme 1999; 122(1): 162-169.

[30] Bertocchi L, Dini D, Giacopini M, et al. Fluid film lubrication in the presence of cavitation: a mass-conserving two-dimensional formulation for compressible, piezoviscous and non-newtonian fluids. Tribol Int 2013; 67: 61-71. 
[31] Salant RF and Fortier AE. Numerical analysis of a slider bearing with a heterogeneous slip/no-slip surface. Tribol T 2004; 47(3): 328-334.

[32] MA GuoJun, WU ChengWei and ZHOU Ping. Hydrodynamics of slip wedge and optimization of surface slip property. Sci China-Phys Mech Astron 2007; (3): 321-330.

[33] Rao TVVLN. Analysis of single-grooved slider and journal bearing with partial slip surface. J Tribol-T Asme 2010; 132(1): 014501.

[34] Oden JT. Applied functional analysis, Upper Saddle River, New Jersey, PrenticeHall, 1979. 


\section{Appendix A: basic properties of the operators $L$ and $M$}

The work reported in [18] demonstrates existence and uniqueness of the problem under examination. In the present paper a formulation based on variational inequalities is developed and coercivity properties are demonstrated for the operator connecting the two complementary variables involved. Such properties allow a numerical routine in terms of linear complementarity to be derived that numerically determines the solution in a finite number of steps.

In this appendix, some basic properties of the operators $L$ and $M$ of equations (6) and (11) are investigated that are fundamental for defining the existence and uniqueness of the solution for the problem under examination.

Symbols $h_{\min }$ and $h_{\max }$ are introduced:

$0<h_{\min } \leq h \leq h_{\text {max }}$

The problem is studied in the functional space $H^{0}(0, a)$ (i.e. $\left.L_{2}(0, a)\right)$ equipped with the norm:

$\|U\|=\left(\int_{0}^{a} U^{2} d x\right)^{\frac{1}{2}}$

and with the inner product:

$(U, V)=\int_{0}^{a} U V d x$

Operator $L$ is self-adjoint, since: 
$(L U, V)=(U, L V)$

Remarkably, it can be shown that under mild conditions operator $L$ is coercive in the following sense:

$(L U, U) \geq \alpha\|U\|^{2} \quad \alpha>0$

In fact:

$(L U, U)=4 \mu \int_{0}^{a} \frac{U^{2}}{h} d x-3 \mu \frac{\left(\int_{0}^{a} \frac{U}{h^{2}} d x\right)^{2}}{\int_{0}^{a} \frac{1}{h^{3}} d x}$

Now, according to the Schwarz inequality [34], the following inequality holds:

$\left(\int_{0}^{a} \frac{U}{h^{2}} d x\right)^{2} \leq \int_{0}^{a} U^{2} d x \int_{0}^{a} \frac{1}{h^{4}} d x$

and therefore: 


$$
\begin{aligned}
& (L U, U) \geq 4 \mu \int_{0}^{a} \frac{U^{2}}{h} d x-3 \mu \int_{0}^{a} U^{2} d x \frac{\int_{0}^{a} \frac{1}{h^{4}} d x}{\int_{0}^{a} \frac{1}{h^{3}} d x}= \\
& =\int_{0}^{a} U^{2}\left[\frac{4 \mu}{h}-3 \mu \frac{\int_{0}^{a} \frac{1}{h^{4}} d y}{\int_{0}^{a} \frac{1}{h^{3}} d y}\right] d x \geq \\
& \geq\left[\frac{4 \mu}{h_{\max }}-3 \mu \frac{\int_{0}^{a} \frac{1}{h^{4}} d x}{\int_{0}^{a} \frac{1}{h^{3}} d x}\right] \int_{0}^{a} U^{2} d x
\end{aligned}
$$

If:

$$
h_{\max } \frac{\int_{0}^{a} \frac{1}{h^{4}} d x}{\int_{0}^{a} \frac{1}{h^{3}} d x}<\frac{4}{3}
$$

the last parenthetical term of equation (A8) is positive, and therefore operator $L$ is coercive.

For a generic profile, positiveness of (A9) can be checked by introducing in it the selected $h$ profile and by computing the corresponding integrals. For instance, in the case of a linear fluid film profile, condition (A9) is satisfied if $h_{\max }$ does not exceed the golden section, $\Phi=(\sqrt{5}+1) / 2 \approx 1.618$, of $h_{\min }$. If, on the other side, no information is available on the shape of $h$ apart from its maximum $\left(h_{\max }\right)$ and minimum $\left(h_{\min }\right)$ values, condition (A9) is still verified if the most pessimistic condition $h_{\max } / h_{\min }<\sqrt[4]{4 / 3}$ is 
satisfied. However, the higher the slope of the slider, the more questionable is the validity of the Reynolds equation. The numerical examples collected in Figure 4 include configurations for which the above inequality is not fulfilled. Encouragingly, the numerical routines are capable of finding the solution even for such cases. It should be noted that in [18] existence and uniqueness of the tangential velocity slip problem are proved without the need to introduce the particular condition on the slider profile $h$ expressed by inequality (A9).

Coerciveness of operator $L$ implies that $L$ is bounded below, that is:

$\|L U\| \geq \alpha\|U\| \quad \alpha>0$

In fact:

$$
\|L U\|\|U\| \geq(L U, U) \geq \alpha\|U\|^{2}
$$

which implies inequality (A10). Consequently $L^{-1}$ exists and is continuous [34].

Interestingly, it can be shown that $L$ is also bounded, that is:

$$
\|L U\| \leq \beta\|U\| \quad \beta>0
$$

In fact:

$$
\|L U\|=\left(\int_{0}^{a}\left[4 \mu \frac{U}{h}-3 \frac{\mu}{h^{2}} \frac{\int_{0}^{a} \frac{U}{h^{2}} d y}{\int_{0}^{a} \frac{1}{h^{3}} d y}\right]^{2} d x\right)^{\frac{1}{2}}
$$

By employing the Minkowski inequality [34], one obtains: 
$\|L U\| \leq 4 \mu\left(\int_{0}^{a} \frac{U^{2}}{h^{2}} d x\right)^{\frac{1}{2}}+3 \mu \frac{\left|\int_{0}^{a} \frac{U}{h^{2}} d x\right|}{\int_{0}^{a} \frac{1}{h^{3}} d x}\left(\int_{0}^{a} \frac{1}{h^{4}} d x\right)^{\frac{1}{2}}$

By employing the Schwarz inequality expressed in (A7), one obtains:

$\|L U\| \leq 4 \mu\left(\int_{0}^{a} \frac{U^{2}}{h^{2}} d x\right)^{\frac{1}{2}}+3 \mu \frac{\int_{0}^{a} \frac{1}{h^{4}} d x}{\int_{0}^{a} \frac{1}{h^{3}} d x}\left(\int_{0}^{a} U^{2} d x\right)^{\frac{1}{2}}$

By employing equation (A7), one obtains:

$\|L U\| \leq\left[\frac{4 \mu}{h_{\max }}+3 \mu \frac{\int_{0}^{a} \frac{1}{h^{4}} d x}{\int_{0}^{a} \frac{1}{h^{3}} d x}\right]\|U\|$

where the term multiplying $\|U\|$ is always positive.

Finally, it can be shown that $L^{-1}$ is coercive according to equation (11). In fact, by putting $V=L U$, one has:

$$
\left(L^{-1} V, V\right)=(L U, U) \geq \alpha\|U\|^{2} \geq \frac{\alpha}{\beta^{2}}\|L U\|^{2}=\frac{\alpha}{\beta^{2}}\|V\|^{2}
$$

It is observed that coerciveness of $L^{-1}$ is demonstrated in equation (A17) by exploiting both coerciveness and boundedness of $L$. Coerciveness of $L^{-1}$ is fundamental for stating the uniqueness of the solution. 


\section{Appendix B: analytical model}

In this appendix, the derivation is presented of the equations for determining the analytical upper and lower bounds of the partial slip zone of Figure 4. The methodology employed is the same as that presented by Spikes in [17] except for the fact that in this case the lubricant is considered slipping against the lower, moving surface, while in [17] slip is assumed to occur against the upper stationary surface of the bearing.

Consider the equilibrium of an orthogonal element of fluid located between two moving, solid surfaces (in this case the upper surface is stationary and the lower one moves with velocity $U^{+}$):

$$
\begin{aligned}
& \frac{\partial \tau_{x z}}{\partial z}=-\frac{\partial p}{\partial x} \\
& \frac{\partial \tau_{y z}}{\partial z}=-\frac{\partial p}{\partial y}
\end{aligned}
$$

where $\tau$ is the shear stress and $p$ is the pressure. For the convention on the sign of the shear stress, $\tau$, reference has to be made to Figure 1. In the current paper, the particular case of a lower moving interface where slip can occur is considered and, at this interface, it is assumed that the fluid experiences a prescribed critical shear stress $\tau_{c x}$ in the $x$ direction, and $\tau_{\mathrm{c} y}$ in the $y$ direction.

If a perfect slip condition is considered, $\tau_{\mathrm{cx}}=\tau_{\mathrm{cy}}=0$.

Equation (B1a) can then be integrated directly assuming $\tau_{\mathrm{xz}}=\tau_{\mathrm{c} x}$ at $z=0$ to yield: 


$$
\tau_{x z}=-\frac{\partial p}{\partial x} z+\tau_{c x}
$$

The expression for the shear stress, $\tau_{\mathrm{xz}}=-\mu \partial \mathrm{u} / \partial \mathrm{z}$, where $\mu$ is the fluid dynamic shear viscosity and $u$ is the fluid velocity, can then be substituted into equation (B2):

$$
-\mu \frac{\partial u}{\partial z}=-\frac{\partial p}{\partial x} z+\tau_{c x}
$$

Assuming that $\mu$ is independent of $z$ and taking the boundary condition at the wetted, no-slip, fixed, upper surface to be $u=0$ at $z=h$, gives on integration:

$$
u=\frac{1}{\mu} \frac{\partial p}{\partial x}\left(\frac{z^{2}}{2}-\frac{h^{2}}{2}\right)-\frac{\tau_{c x}}{\mu}(z-h)
$$

A similar expression can be derived for the $y$ direction.

The fluid velocity at the slipping surface in the $x$ direction, $U$, is obtained from equation (B4) by substituting $z=0$ :

$U=-\frac{h^{2}}{2 \mu} \frac{\partial p}{\partial x}+\frac{\tau_{c x}}{\mu} h$

Equation (B4) is integrated across the film thickness to yield the flow though a column of fluid, e.g. for flow in the $x$ direction:

$$
q_{x}=-\frac{h^{3}}{3 \mu} \frac{\partial p}{\partial x}+\frac{\tau_{c x} h^{2}}{2 \mu}
$$

From this, the flow differential in the $x$ direction is: 


$$
\frac{\partial q_{x}}{\partial x}=-\frac{\partial}{\partial x}\left(\frac{h^{3}}{3 \mu} \frac{\partial p}{\partial x}\right)+\frac{\partial}{\partial x}\left(\frac{\tau_{c x} h^{2}}{2 \mu}\right)
$$

with a similar expression in the $y$ direction.

On the basis of continuity of flow and assuming no supply of lubricant in the $z$ direction:

$$
\frac{\partial q_{x}}{\partial x}+\frac{\partial q_{y}}{\partial y}=0
$$

Substituting equation (B7) and its analogue in the $y$ direction into equation (B8) and rearranging one obtains:

$$
\frac{\partial}{\partial x}\left(\frac{h^{3}}{3 \mu} \frac{\partial p}{\partial x}\right)+\frac{\partial}{\partial y}\left(\frac{h^{3}}{3 \mu} \frac{\partial p}{\partial y}\right)=\frac{\partial}{\partial x}\left(\frac{\tau_{c x} h^{2}}{2 \mu}\right)+\frac{\partial}{\partial y}\left(\frac{\tau_{c y} h^{2}}{2 \mu}\right)
$$

For an infinitely long bearing, $\partial p / \partial y=0$ and $\partial h / \partial y=0$ and assuming that viscosity does not vary over the bearing area, i.e. $\mu$ is independent of $x$, the equation governing the slip problem can be finally derived:

$$
\frac{d}{d x}\left(h^{3} \frac{d p}{\partial x}\right)=\frac{3 \tau_{c}}{2} \frac{d}{d x}\left(h^{2}\right)
$$

Consider now the classical one-dimensional Reynolds equation (1):

$$
\frac{d}{d x}\left(h^{3} \frac{d p}{\partial x}\right)=6 U \mu \frac{d h}{d x}
$$


The right hand sides of equations (B10) and (B11) look quite different. In particular, in equation (B10) the sliding velocity $U$ does not appear. Thus, for the limit condition $\tau_{\mathrm{c}}=$ 0 , no lubricant entrains the bearing profile and thus no load support exists.

Consider then the non-dimensional form of equation (B10):

$\frac{d}{d x^{*}}\left(h^{* 3} \frac{d p^{*}}{\partial x^{*}}\right)=\frac{3 \tau_{c}^{*}}{2} \frac{d}{d x^{*}}\left(h^{* 2}\right)$

where the non-dimensional film shape is $h^{*}=1+K-K x^{*}$.

Integrating twice equation (B12) and imposing proper boundary conditions at the bearing extremities,

$\left.p^{*}\right|_{x^{*}=0}=\left.p^{*}\right|_{x^{*}=1}=0$

the non-dimensional expression of the pressure distribution $p^{*}$ can be derived:

$$
p^{*}=\tau_{c}^{*}\left[\frac{3}{2} \frac{\ln (K+1)(K+1)^{2}}{K^{2}(K+2)}-\frac{3}{2} \frac{\ln (K+1)(K+1)^{2}}{K^{2}(K+2) h^{* 2}}-\frac{3}{2} \frac{\ln h^{*}}{K}\right]
$$

Consider now the non-dimensional form of equation (B5):

$$
U^{*}=-\frac{h^{* 2}}{2} \frac{\partial p^{*}}{\partial x^{*}}+\tau_{c}^{*} h^{*}
$$

On one side, when a full slip condition exists, everywhere inside the bearing $U^{*} \leq 1$ (i.e. $U \leq U^{+}$) or $U^{*} \geq 1$ (i.e. $U \geq U^{+}$). Therefore, in order to determine the lower analytical bound of the partial slip zone of Figure 4, the following expressions must be considered: 
$-\frac{h^{* 2}}{2} \frac{\partial p^{*}}{\partial x^{*}}+\tau_{c}^{*} h^{*} \leq 1$

or

$-\frac{h^{* 2}}{2} \frac{\partial p^{*}}{\partial x^{*}}+\tau_{c}^{*} h^{*} \geq 1$

For both equations (B16a) and (B16b) the transition between a full slip and a partial slip state can be obtained considering the limit condition:

$-\frac{h^{* 2}}{2} \frac{\partial p^{*}}{\partial x^{*}}+\tau_{c}^{*} h^{*}=1$

Substituting equation (B14) into equation (B17) and solving with respect to $\tau_{c}^{*}$ one obtains:

$$
\tau_{c}^{*}=\frac{4 K h^{*}(K+2)}{K h^{* 2}(K+2)+6 \ln (K+1)(K+1)^{2}}
$$

For $K \geq 0$, i.e. converging bearings, the right hand side of equation (B18) is positive and so, at least with the boundary conditions (B13), the transition between full slip and partial slip only occurs for positive values of $\tau_{c}^{*}$. (The negative values of the shear stress $\tau$ visible in Figure 3 refer to partial slip cases, whereas full slip condition is possible only for positive value of $\tau$, see Figure 2).

In particular, the transition value of $\tau_{c}^{*}$ as a function of $K$ can be detected by finding: 


$$
\min \left(\tau_{c}^{*}, 0 \leq x^{*} \leq 1\right)=\min \left(\frac{4 K h^{*}(K+2)}{K h^{* 2}(K+2)+6 \ln (K+1)(K+1)^{2}}, 0 \leq x^{*} \leq 1\right)
$$

From equation (B19) one obtains:

$$
\min \left(\tau_{c}^{*}, 0 \leq x^{*} \leq 1\right)=\left\{\begin{array}{l}
\left.\tau_{c}^{*}\right|_{x^{*}=1}, K \leq \underline{K} \\
\left.\tau_{c}^{*}\right|_{x^{*}=0}, K>\underline{K}
\end{array}\right.
$$

where $\underline{K} \cong 16$ is the solution of the following equation:

$$
6 \ln (K+1)(K+1)-K(K+2)=0
$$

Substituting equation (B18) into equation (B20) the analytical definition of the frontier between full slip and partial slip conditions can be finally detected:

$$
\tau_{c}^{*}=\left\{\begin{array}{c}
\frac{4 K(K+2)}{K(K+2)+6 \ln (K+1)(K+1)^{2}}, K \leq \underline{K} \\
\frac{4 K(K+2)}{K(K+1)(K+2)+6 \ln (K+1)(K+1)}, K>\underline{K}
\end{array}\right.
$$

On the other side, when a no-slip condition exists, the problem is described by the classical Reynolds equation with the non-dimensional form of the pressure profile:

$$
p^{*}=\frac{6 K x^{*}\left(x^{*}-1\right)}{h^{* 2}(K+2)}
$$

If no slippage occurs, everywhere inside the bearing domain:

$$
\left|\tau \|_{z=0} \leq \tau_{c} \Rightarrow\right| \frac{h}{2} \frac{d p}{d x}+U \frac{\mu}{h} \mid \leq \tau_{c}
$$


and in a non-dimensional form:

$\left|\tau^{*}\right|=\left|\frac{h^{*}}{2} \frac{d p^{*}}{d x^{*}}+\frac{1}{h^{*}}\right| \leq \tau_{c}^{*}$

In particular, the boundary between the no-slip and the partial slip areas can be detected solving the equation:

$$
\max \left(\left|\tau^{*}\right|, 0 \leq x^{*} \leq 1\right)=\max \left(\left|\frac{h^{*}}{2} \frac{d p^{*}}{d x^{*}}+\frac{1}{h^{*}}\right|, 0 \leq x^{*} \leq 1\right)=\tau_{c}^{*}
$$

Deriving the first term of equation (B24) one obtains:

$$
\max \left(\left|\tau^{*}\right|, 0 \leq x^{*} \leq 1\right)=\left\{\begin{array}{c}
\mid \tau^{*} \|_{x^{*}=0}, K \leq 1 \\
\mid \tau^{*} \|_{x^{*}=\underline{x}^{*}}, 1<K<\underline{K} \\
\mid \tau^{*} \|_{x^{*}=1}, K \geq \underline{K}
\end{array}\right.
$$

where

$$
\underline{x}^{*}=\frac{\left(K^{2}-1\right)}{K(K+2)}
$$

and

$$
\underline{K}=1+\frac{3}{2} \sqrt{2}
$$

Substituting equation (B27) into equation (B26) and considering equation (B23), the analytical definition of the frontier between no-slip and partial-slip conditions can be finally detected: 


$$
\tau_{c}^{*}=\left\{\begin{array}{c}
\frac{2(2 K+1)}{(K+1)(K+2)}, K \leq 1 \\
\frac{2(K+2)}{3(K+1)}, 1<K<\bar{K} \\
\frac{2(K-1)}{K+2}, K \geq \bar{K}
\end{array}\right.
$$

Figure 5 plots the analytical upper and lower bounds of the partial-slip zone of Figure 4 expressed by equations (B22) and (B30). The results of Figure 5 essentially coincide with the numerical results of Figure 4. The dotted line in Figure 5 shows the upper bound of the partial slip zone if only backwards slippage, i.e. with $U \leq U^{+}$, is taken into account.

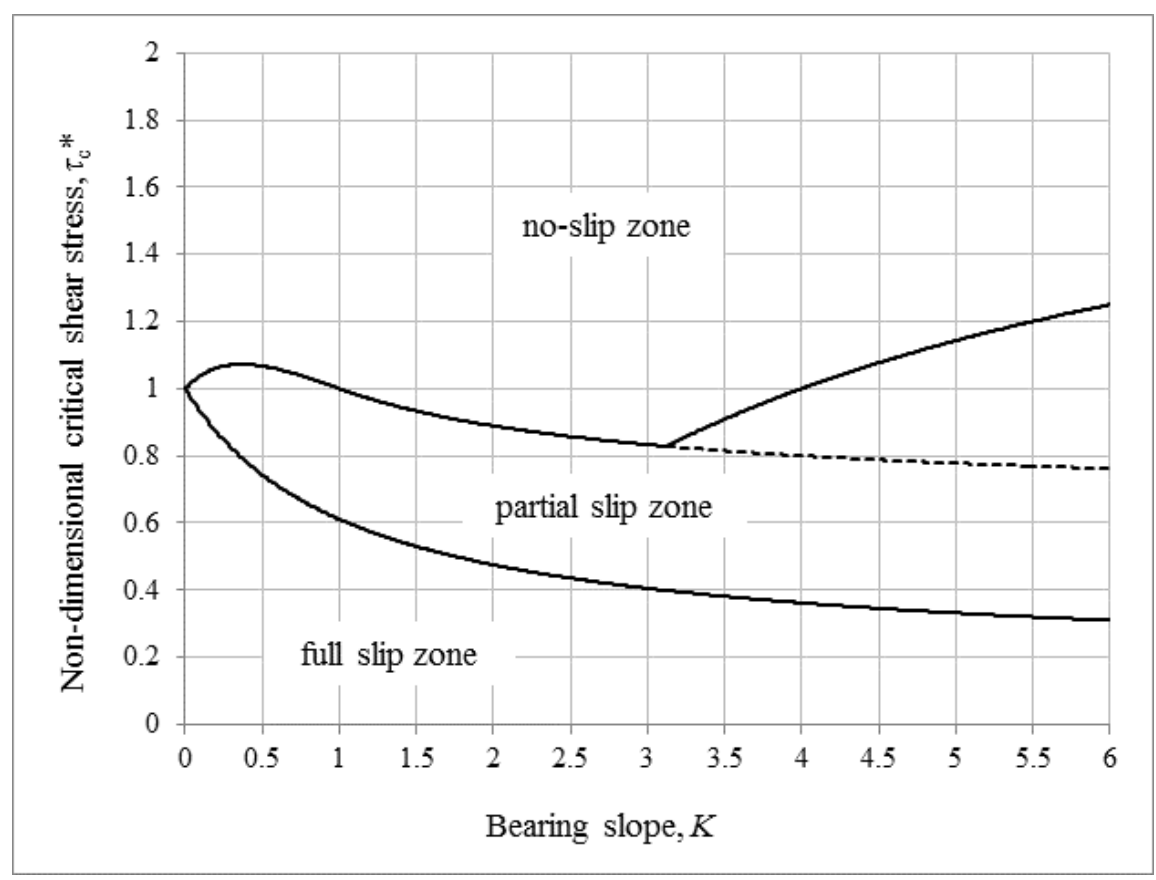

Figure 5. Analytical upper and lower bounds of the partial-slip zone. 


\section{TABLES}

Table 1. Geometrical and physical parameters for the first set of examples.

\begin{tabular}{|l|l|l|}
\hline Film thickness at the inlet $h_{\text {in }}$ & $2 \cdot 10^{-3}$ & $\mathrm{~m}$ \\
\hline Film thickness at the outlet $h_{\text {out }}$ & $1 \mathrm{e}-3$ & $\mathrm{~m}$ \\
\hline Bearing slope $K=h_{\text {in }} / h_{\text {out }}-1$ & 1 & \\
\hline Slider length $a$ & 1 & $\mathrm{~m}$ \\
\hline Fluid pressure at the inlet $p_{0}$ & 0 & $\mathrm{~Pa}$ \\
\hline Fluid pressure at the outlet $p_{a}$ & 0 & $\mathrm{~Pa}$ \\
\hline Sliding velocity $U^{+}$ & 0.4 & $\mathrm{~m} / \mathrm{s}$ \\
\hline Lubricant viscosity $\mu$ & $125 \cdot 10^{-3}$ & $\mathrm{~Pa} \mathrm{~s}$ \\
\hline Critical shear stress $\tau_{\mathrm{c}}$ & $30 / 40 / 50$ & $\mathrm{~Pa}$ \\
\hline
\end{tabular}

Table 2. Geometrical and physical parameters for the second set of examples.

\begin{tabular}{|l|l|l|}
\hline Film thickness at the inlet $h_{\text {in }}$ & $6 \cdot 10^{-3}$ & $\mathrm{~m}$ \\
\hline Film thickness at the outlet $h_{\text {out }}$ & $1 \mathrm{e}-3$ & $\mathrm{~m}$ \\
\hline Bearing slope $K=h_{\text {in }} / h_{\text {out }}-1$ & 1 & \\
\hline Slider length $a$ & 1 & $\mathrm{~m}$ \\
\hline Fluid pressure at the inlet $p_{0}$ & 0 & $\mathrm{~Pa}$ \\
\hline Fluid pressure at the outlet $p_{a}$ & 0 & $\mathrm{~Pa}$ \\
\hline Sliding velocity $U^{+}$ & 0.1 & $\mathrm{~m} / \mathrm{s}$ \\
\hline Lubricant viscosity $\mu$ & $125 \cdot 10^{-3}$ & $\mathrm{~Pa} \mathrm{~s}$ \\
\hline Critical shear stress $\tau_{\mathrm{c}}$ & $8 / 10 / 15$ & $\mathrm{~Pa}$ \\
\hline
\end{tabular}




\section{FIGURES}

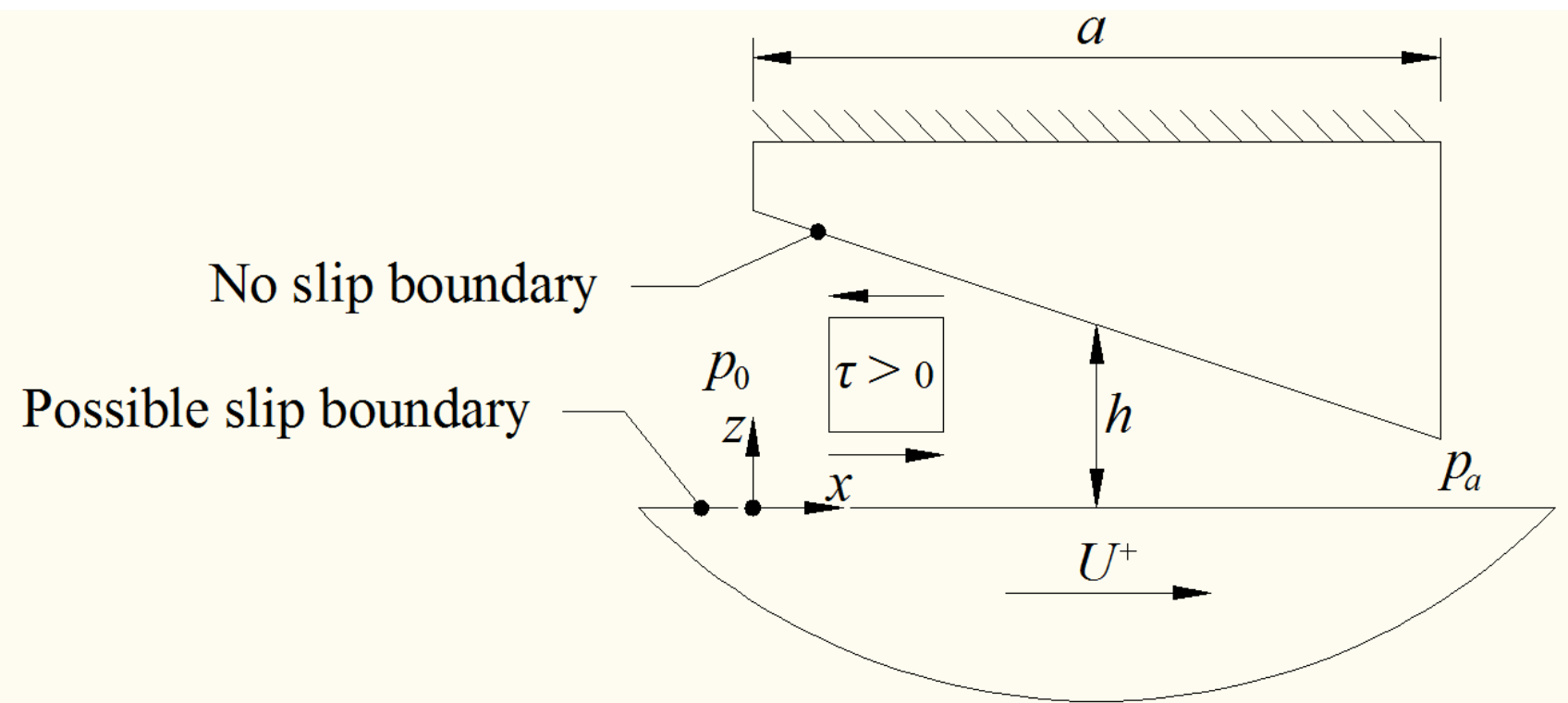

Figure 1. The meaning of main symbols adopted for the tangential velocity slip problem. 


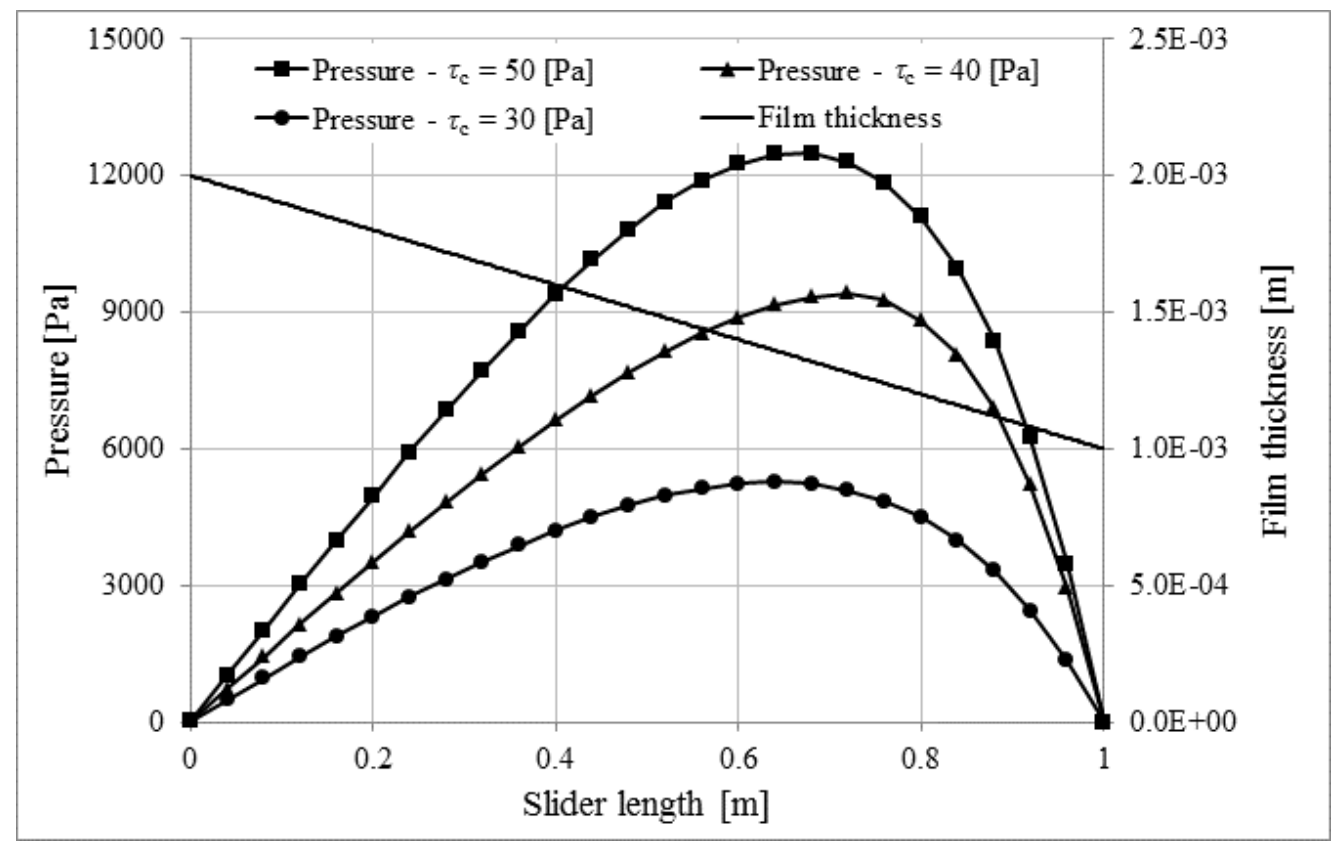

(a)

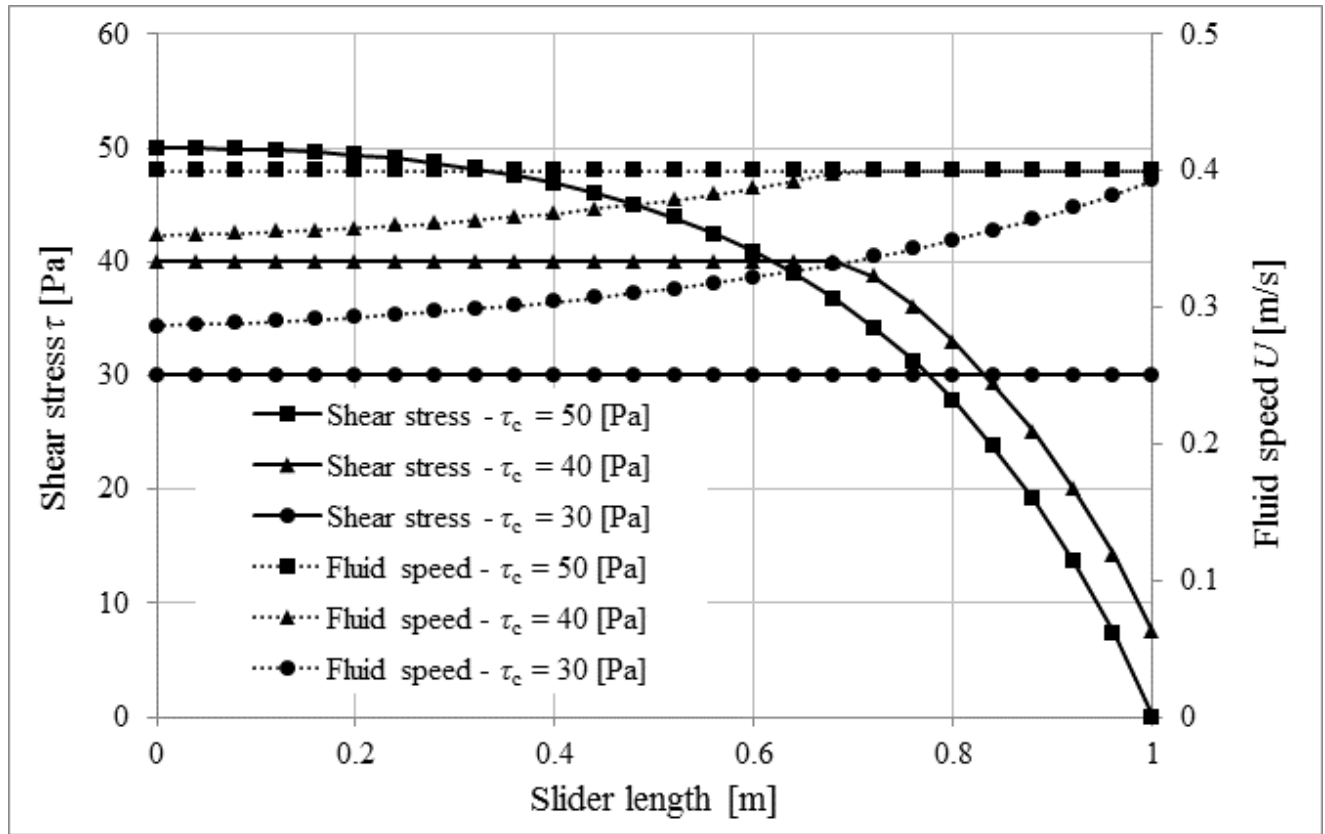

(b)

Figure 2. Fluid pressure (a), shear stress and fluid layer speed (b), for the selected cases of Table 1. 


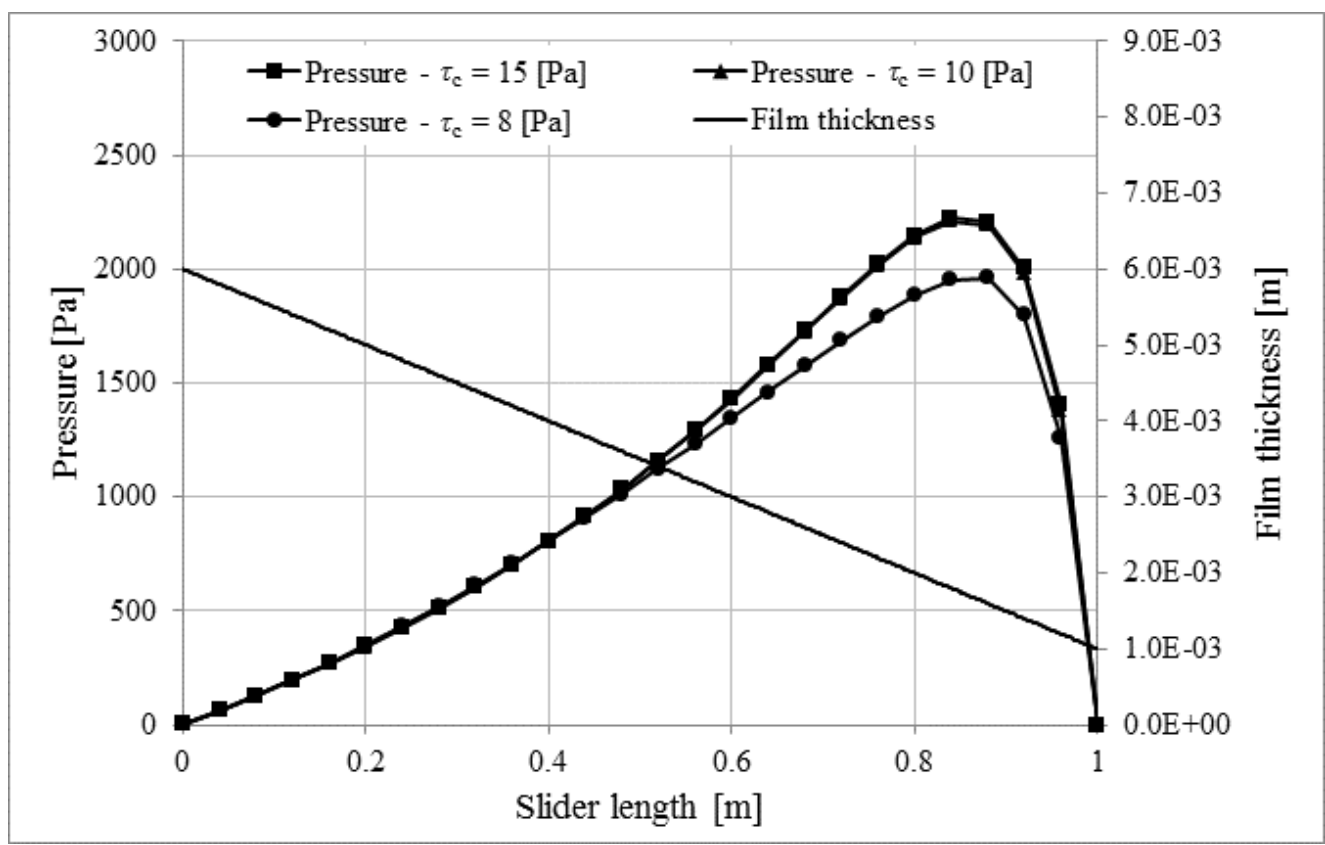

(a)

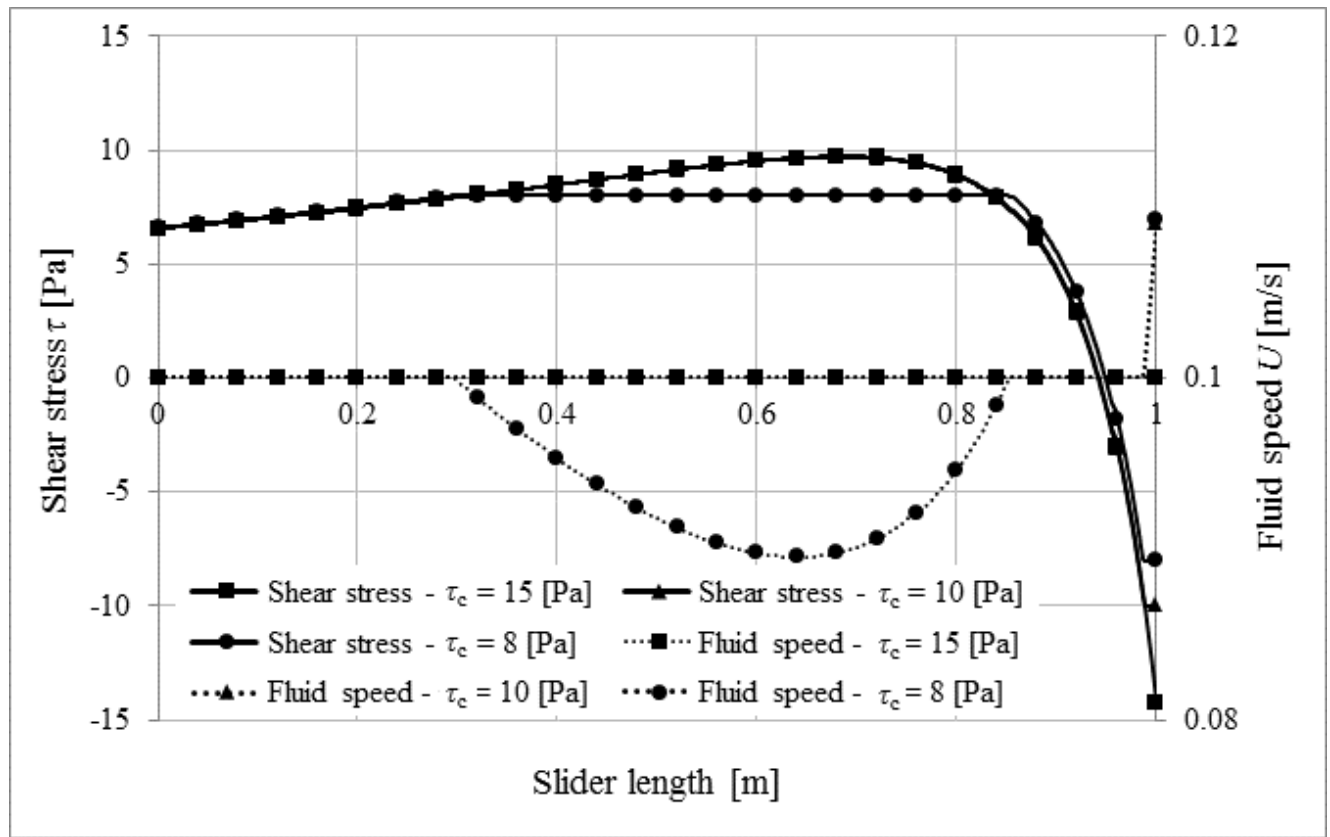

(b)

Figure 3. Fluid pressure (a), shear stress and fluid layer speed (b), for the selected cases of Table 2. 


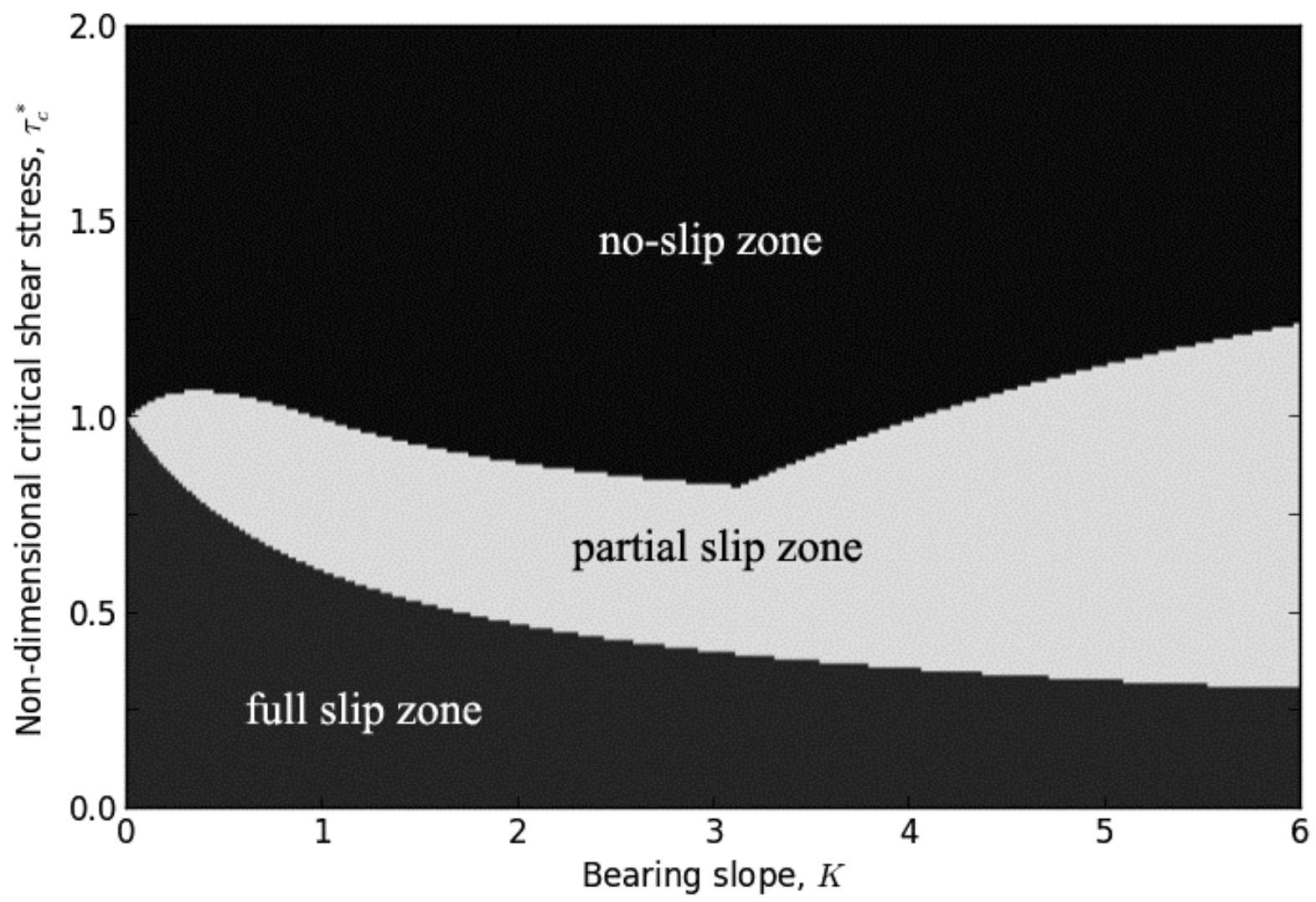

Figure 4. Map of the contact slip conditions in a convergent slider. 


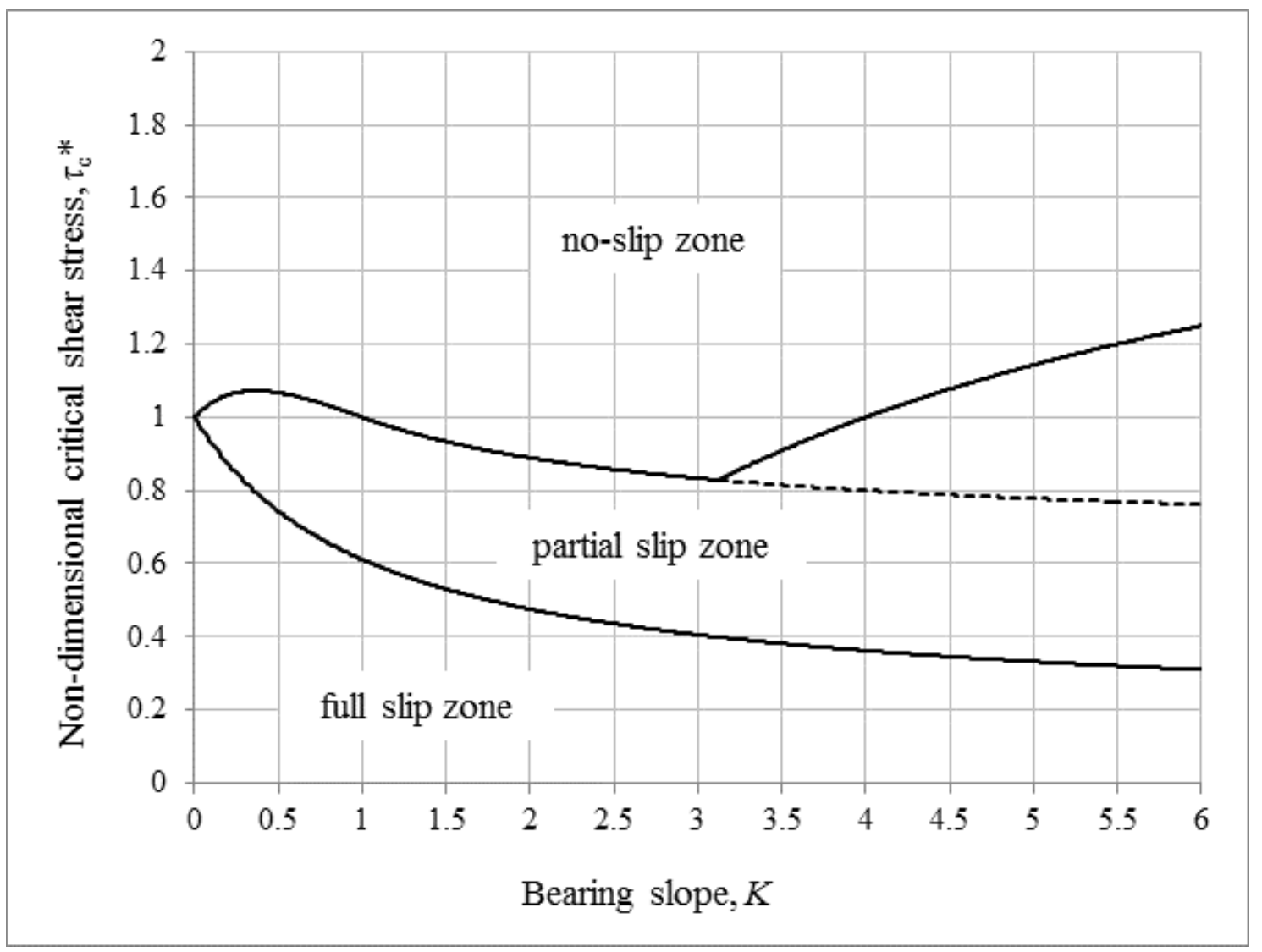

Figure 5. Analytical upper and lower bounds of the partial slip zone. 